\title{
H3K27 Acetylation of SF1 in PBMC: A Biomarker for Adrenal Insufficiency of Steroid Synthesis in Male Offspring Induced by Prenatal Dexamethasone Exposure
}

\section{Guanghui Chen}

Wuhan University

Can Ai

Wuhan University

Fangfang Duan

Wuhan University

Yawen Chen

Wuhan University

Jiangang Cao

Wuhan University

Jinzhi Zhang

Wuhan University

Ying Ao

Wuhan University

Hui Wang ( $\square$ wanghui19@whu.edu.cn )

Wuhan University

\section{Research Article}

Keywords: prenatal dexamethasone exposure, adrenal insufficiency, histone acetylation, intrauterine programming, warning marker

Posted Date: June 18th, 2021

DOI: https://doi.org/10.21203/rs.3.rs-581938/v1

License: (c) (i) This work is licensed under a Creative Commons Attribution 4.0 International License. Read Full License 


\section{Abstract}

Dexamethasone is widely used for treating pregnant diseases. However, lots of studies have confirmed that prenatal dexamethasone exposure (PDE) could increase the risk of offspring multiple diseases. The purpose of this study was to elucidate the intrauterine epigenetic mechanism of adrenal developmental programming, and to explore early warning marker in peripheral blood mononuclear cells (PBMC). We found the adrenal morphological and functional changes before and after birth in PDE male offspring rats, which were performed as the decreases of serum corticosterone concentration, steroidogenic acute regulatory (StAR) expression, histone 3 lysine 27 acetylation (H3K27ac) level of steroidogenic factor1 (SF1) promoter region and its expression; meanwhile, the expression of glucocorticoid receptor (GR) and histone acetylation enzyme 5 (HDAC5) in PDE male fetal rats were increased. In vitro, dexamethasone reduced the expression of SF1 and StAR, and cortisol production, but increased the expression of GR and HDAC5, the binding between GR and SF1 promoter region, and protein interaction between GR and HDAC5. GR siRNA or HDAC5 siRNA could reverse the above effects of dexamethasone. Furthermore, in vivo, we confirmed that H3K27ac level of SF1 promoter region and its expression in PBMC of PDE group were decreased before and after birth, showing a positive correlation with the same indexes in adrenal. Meanwhile, in clinical trials, we confirmed that H3K27ac level of SF1 promoter region and its expression were decreased after prenatal dexamethasone application in neonatal PBMC. In conclusion, PDE induced adrenal insufficiency of male offspring rats, which was related to activate adrenal GR by dexamethasone in uterus. The activated GR, on one hand, increased its direct binding to SF1 promoter region to inhibit its expression, on the other hand, up-regulated and recruited HDAC5 to decrease H3K27ac level of SF1 promoter region, and strengthened the inhibition of SF1 and subsequent StAR expression.

\section{Introduction}

World Health Organization estimates that global premature birth rates are $10.6 \%$ and about 14.84 million premature babies are born each year ${ }^{[1]}$. As a synthetic glucocorticoid, dexamethasone penetrates the placenta easily and promotes fetal liver maturation, is widely used in pregnant women with premature birth risk for improving the survival rate of premature infants ${ }^{[2-4]}$. However, prenatal dexamethasone treatment is a double-edged sword. Numerous studies have shown that dexamethasone has fetal developmental toxicity, which could cause low birth weight and susceptibility to various diseases in adulthood ${ }^{[5,6]}$. In addition, researches also have discovered that prenatal dexamethasone exposure (PDE) affected adrenal function in offspring mice ${ }^{[7,8]}$. These results suggest that dexamethasone has adrenal developmental toxicity, but the potential mechanisms are unclear.

Adrenal is an important endocrine organ, which takes cholesterol as raw material to synthesize glucocorticoids, which plays an important role in regulating metabolic homeostasis and normal development. The adrenal steroid synthesis systems include steroidogenic acute regulatory protein (StAR),

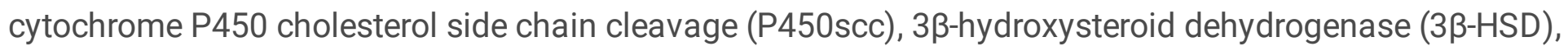
steroid 21-hydroxylase (P450c21), and steroid 11ß-hydroxylase (P450c11) [9]. Steroidogenic factor1 (SF1), a nuclear receptor expressed in steroidogenic tissues, a common transcription factor for steroid synthesis 
systems [10-11]. "Intrauterine programming" refers to that the structure and function of tissues are changed permanently by adverse environment during early life stage, and can continue after birth ${ }^{[12]}$. Epigenetics means the inherited changes in biological phenotype or gene expression pattern without any alteration in DNA sequence, including histone acetylation ${ }^{[13-15]}$. We hypothesized that PDE induced adrenal insufficiency in adult offspring rats may be associated with intrauterine epigenetic programming alteration of SF1.

Some studies suggest that offspring's adrenal dysfunction caused by adverse pregnancy environment is related to susceptibility to metabolic syndrome in adults ${ }^{[16,17]}$. Our previous studies have confirmed that prenatal caffeine exposure could induce adrenal dysplasia in offspring rats, characterized by glucocorticoid-dependence peripheral glucose and lipid metabolic function changes ${ }^{[16,17]}$. Therefore, early warning of fetal-originated adrenal dysfunction will be helpful to prevent and cure the occurrence of metabolic syndrome in adults. However, early warning of fetal-originated adrenal dysfunction is still difficult for lack of accurate and sensitive biomarker and risk assessment system. Biomarkers are indicators that could be measured objectively and evaluated normal biological and pathological process [18]. The blood samples, including peripheral blood monocytes (PBMC), could be easily obtained in clinic, the detection of blood biomarkers can achieve the characteristics of sampling and analysis at any time, no hysteresis and good repeatability, which has shown a strong application prospect in early diagnosis, efficacy monitoring and complication risk assessment ${ }^{[19,20]}$. The researches suggest that epigenetic modifications of PBMC are often used as biomarkers for early diagnosis of tumor ${ }^{[21,22]}$. So, are there any specific biomarkers in the blood for adrenal insufficiency of offspring induced by PDE in the early life?

In this study, we first confirmed intrauterine programming alteration of adrenal insufficiency in PDE offspring by observing effects of PDE on adrenal morphology and function before and after birth. Further, the epigenetic mechanism and toxicity target of adrenal insufficiency induced by dexamethasone were elucidated in vivo and in vitro. Finally, PBMC from PDE offspring rats and clinical neonates were collected and tested, and the correlations were analyzed to verify the peripheral blood warning marker of adrenal insufficiency in PDE offspring. This study will provide an important experimental and theoretical basis for interpreting fetal-originated diseases, guiding rational drug use during pregnancy and the early prevention and treatment of fetal-originated diseases.

\section{Materials And Methods}

\subsection{Chemicals and reagents}

Dexamethasone (in ampoules) was purchased from Shuanghe Pharmaceutical Company (Wuhan, China). Isoflurane was obtained from Baxter Healthcare Co. (Deerfield, IL, USA). Rat corticosterone and human cortisol enzyme-linked immunosorbent assay (ELISA) kits were obtained from Assay pro LLC. (Saint Charles, MO, USA). BCA Assay Kit was from Sigma-Aldrich Co., Ltd. (St Louis, MO, USA). The antibodies of StAR (ab203193), SF1 (ab65815), histone deacetylase 5 (HDAC5) (ab55403) and glucocorticoids receptor (GR) (ab183127) were purchased from AbcamCo., Ltd. (Cambridge, UK). Acetyl histone 3 lysine 9 (H3K9ac) 
(A7255), H3K14ac (A7254), H3K27ac (A7253) goat anti-rabbit IgG (AC005) were purchased from ABclonal Technology Co., Ltd. (Wuhan, China). DNA purification kit (Q5314) was purchased from TIANGEN Biotech Co., Ltd. (Beijing, China). GR siRNA and HDAC5 siRNA sequences were synthesized by Gene Pharma (Shanghai, China). Trizol reagent kit was obtained from Omega Bio-Tek (Doraville, Georgia USA). Reverse transcription and real-time quantitative polymerase chain reaction (RT-qPCR) kits were purchased from SYBR GREEN Biotechnology Co., Ltd. (Dalian, China). All the oligonucleotide primers were synthesized by Sangon Biotech Co., Ltd. (Shanghai, China). Other chemicals and agents were of analytical grade.

\subsection{Animals and treatment}

Animal experiments performed in the present study were approved by the Animal Welfare Committee at Wuhan University (Wuhan, China), and in accordance with the Guidelines for the Care and Use of Laboratory Animals at Wuhan University (No.14016). Specific pathogen-free Wistar rats [No. 2012-2014, certification number: 42000600002258, license number: SCXK(Hubei)], weighing 180-220 g (female) and 260-300 g (male), were obtained from the Experimental Center of the Hubei Medical Scientific Academy (Wuhan, China). After 1 week of accommodation, one male and two female rats were placed together overnight for mating. Gestational day (GD) 0 was determined upon confirmation of mating by the appearance of sperm in a vaginal smear. Pregnant rats were transferred to individual cages and then randomly divided into control, $\operatorname{PDE}(\mathrm{L})$ and $\operatorname{PDE}(\mathrm{H})$ groups. From $\mathrm{GD} 9$ to $\mathrm{GD} 20$, the $\operatorname{PDE}(\mathrm{L})$ and $\operatorname{PDE}(\mathrm{H})$ group were subcutaneously injected with 0.2 and $0.8 \mathrm{mg} / \mathrm{kg}$.d dexamethasone, and the control group received the same volume of the distilled water.

On GD20, part of pregnant rats was anesthetized with $3 \%$ isoflurane and then sacrificed, fetuses were weighed after being dried on filter papers. Pregnant rats with litter sizes of 12 to 14 were considered qualified. Briefly, fetal blood samples were collected, serum and PBMC were isolated, and fetal adrenals were collected. The samples collected from littermates were pooled, immediately frozen in liquid nitrogen, and stored at $-80^{\circ} \mathrm{C}$ for subsequent analyses. The remaining pregnant rats in control and $\mathrm{PDE}(\mathrm{L})$ groups were allowed to delivery normally. The number of pregnant rats with the litter size of 8-14 at birth was assigned to 12 ( 6 male pups and 6 female pups) for each group. After weaning on postnatal week (PW) 4, the offspring rats were given normal diet. On PW6, PW8 and PW28, the rats were anesthetized with $3 \%$ isoflurane in a room separated from others, the sample collection was similar to that in utero.

\subsection{Serum corticosterone and cell medium cortisol concentration measurements}

The concentration of serum corticosterone and cell medium cortisol were measured by ELISA kits, following the manufacturer's protocols. The intra-assay and inter-assay coefficients of variation for corticosterone and cortisol determination were $5.0 \%$ and $7.2 \%$, respectively.

\subsection{Total RNA extraction and RT-qPCR}

Total RNA was isolated from offspring rat adrenals using Trizol reagent according to the manufacturer's protocol. For RT-qPCR analysis, single-strand cDNA was prepared from $1 \mathrm{~g}$ of total RNA according to the protocol of the Exscript RT reagent kit. Primers were designed using Primer Premier 5.0, and their 
sequences are shown in Table 1. PCR assays were performed in 96-well optical reaction plates using the $A B I$ Step real-time PCR thermal cycler (ABI Stepone, NY, USA). To precisely quantify gene transcripts, the mRNA level of the housekeeping gene glyceraldehyde phosphate dehydrogenase (GAPDH) was measured as the quantitative control, and each sample was normalized to the GAPDH mRNA content. PCR cycling conditions were as follows: pre-denaturation, $95^{\circ} \mathrm{C}$ for $30 \mathrm{~s}$; denaturation, $95^{\circ} \mathrm{C}$ for $5 \mathrm{~s}$; gene-specific annealing conditions were listed in Supplemental Tables 1 and $72^{\circ} \mathrm{C}$ for $30 \mathrm{~s}$ for elongation.

Table 1

Oligonucleotide primers and conditions of real-time quantitative polymerase chain reaction (RT-PCR).

\begin{tabular}{|lllll|}
\hline Genes & Forward primer & Reverse primer & $\begin{array}{l}\text { Product } \\
\text { (bp) }\end{array}$ & Annealing \\
\hline SF1 & CCAGTACGGCAAGGAAGA & GAGGCTGAAGAGGATGAGGA & 193 & $63^{\circ} \mathrm{C}, 30 \mathrm{~s}$ \\
\hline StAR & GGGAGATGCCTGAGCAAAGC & GCTGGCGAACTCTATCTGGGT & 188 & $65^{\circ} \mathrm{C}, 30 \mathrm{~s}$ \\
\hline P450scc & GCTGCCTGGGATGTGATTTTC & GATGTTGGCCTGGATGTTCTTG & 156 & $63^{\circ} \mathrm{C}, 30 \mathrm{~s}$ \\
\hline P450c21 & AGGAGCTGCAGCACAGTTGAC & ATACCCTTATTTTTGAGGGC & 271 & $58^{\circ} \mathrm{C}, 30 \mathrm{~s}$ \\
\hline P450c11 & CCCCTTTGTGGATGTGGTAG & CACGCTCTCAGGTTTCAGGT & 190 & $61^{\circ} \mathrm{C}, 30 \mathrm{~s}$ \\
\hline GR & CACCCATGACCCTGTCAGTC & AAAGCCTCCCTCTGCTAACC & 156 & $63^{\circ} \mathrm{C}, 30 \mathrm{~s}$ \\
\hline $\begin{array}{l}\text { HDAC5- } \\
\text { R }\end{array}$ & CCCATTGGAGATGTGGAATAC & TCTGGCGGTGACAGAATA & 210 & $61^{\circ} \mathrm{C}, 30 \mathrm{~s}$ \\
\hline $\begin{array}{l}\text { GAPDH- } \\
\text { R }\end{array}$ & GCAAGTTCAATGGCACAG & GCCAGTAGACTCCACGACA & 140 & $63^{\circ} \mathrm{C}, 30 \mathrm{~s}$ \\
\hline GR-H & TTCTGACTGGGGCCAATGAA & AATGATGGTGGCCTCGAGA & 151 & $60^{\circ} \mathrm{C}, 30 \mathrm{~s}$ \\
\hline StAR-H & ACAGGTACGAGAGGGATGCT & CCATCACTCTCCAGCCCTTG & 234 & $60^{\circ} \mathrm{C}, 30 \mathrm{~s}$ \\
\hline $\begin{array}{l}\text { HDAC5- } \\
\text { H }\end{array}$ & CTCTGTGCTCTACATCTCTCT & TGTAAGGTACTCCACGTCTC & 185 & $60^{\circ} \mathrm{C}, 30 \mathrm{~s}$ \\
\hline SF1-H & CCCAGGTACCCTTCTCCCAG & TGCCGAGCTGTAAAACCCAA & 316 & $61^{\circ} \mathrm{C}, 30 \mathrm{~s}$ \\
\hline $\begin{array}{l}\text { GAPDH- } \\
\text { H }\end{array}$ & ACGAGCCCACATTTCCCAAT & TGAGAATGGCTGCTCCCTTG & 186 & $60^{\circ} \mathrm{C}, 30 \mathrm{~s}$ \\
\hline
\end{tabular}

SF1: steroidogenic factor 1; StAR: steroidogenic acute regulatory; P450scc: cytochrome P450 cholesterol side-chain cleavage; $3 \beta \mathrm{HSD}$ : $3 \beta$-hydroxysteroid dehydrogenase; P450c21: steroid 21-hydroxylase; P450c11: steroid 11ß-hydroxylase; GR: glucocorticoids receptor; HDAC5: histone deacetylase 5; GAPDH: glyceraldehyde 3-phosphate dehydrogenase. $\mathrm{R}$ : rat; $\mathrm{H}$ : human. 
Table 2

Human and rat primers used for Chromatin immunoprecipitation-polymerase chain reaction (ChIP-PCR).

\begin{tabular}{|llll|}
\hline Genes & Forward primer $\left(5^{\prime} \mathbf{3}^{\prime}\right)$ & Reverse primer $\left(5^{\prime} \mathbf{3}^{\prime}\right)$ & Annealing \\
\hline SF1 (human) & CTGCGCGCCATTCTCCAGC & TACGTGGTCGGGTGATCATGA & $\begin{array}{l}60^{\circ} \mathrm{C}, 30 \\
\mathrm{~s}\end{array}$ \\
\hline SF1 (rat) & CAGTGTATGTGATGCTGGGG & GAGTCTGAAGAGCTGAGGTC & $\begin{array}{l}60^{\circ} \mathrm{C}, 30 \\
\mathrm{~S}\end{array}$ \\
\hline $\begin{array}{l}\text { GR-SF1 } \\
\text { binding(human) }\end{array}$ & ACGCCATTTATCAACGAAATCA & TGCAACAGAATCCGGAAATACC & $\begin{array}{l}60^{\circ} \mathrm{C}, 30 \\
\mathrm{~s}\end{array}$ \\
\hline
\end{tabular}

SF1: steroidogenic factor 1, GR: glucocorticoids receptor.

\subsection{Histological and immunohistochemical analysis}

Adrenals were fixed in $4 \%$ paraformaldehyde overnight and processed with the paraffin sectioning technique. $5 \mu \mathrm{m}$-thick slices were prepared and routinely stained with hematoxylin-eosin (H\&E). Every 5th section of the series was saved, observed and photographed with an Olympus AH-2 light microscope (Olympus, Tokyo, Japan). On these sections, the maximal cross-sectional areas of adrenal were determined.

To observe the specific protein expression, adrenals were fixed in 4\% paraformaldehyde for $24 \mathrm{~h}$, embedded with paraffin. Samples were cut into $5 \mu \mathrm{m}$-thick slices along the longitudinal axis. The immunohistochemical procedures were performed using a streptavidin-peroxidase (SP)-conjugated method according to the manufacturer's instructions. Briefly, $3 \% \mathrm{H}_{2} \mathrm{O}_{2}$ was added for 10 min to block endogenous peroxidase activity, and pre-immune goat serum was used to block the non-specific binding sites. The sections were then incubated at $37^{\circ} \mathrm{C}$ for $20 \mathrm{~min}$ with an anti-StAR antibody (1:400), anti-SF1 antibody (1:300), anti-HDAC5 antibody (1:500), or anti-GR antibody (1:500). For a negative staining control, PBS was used in place of the antibodies. The signal was visualized using light microscopy, imaged and the positively stained areas were analyzed using the Photo Imaging System (HMIAS-2000, Guangzhou, China).

\subsection{Cell culture and treatment}

Human adrenocortical H295R cells were seeded in a culture medium (DMEM/F12 medium with 10\% fetal bovine serum, $1 \%$ streptomycin and penicillin) at $37^{\circ} \mathrm{C}$ in an atmosphere of $95 \%$ air and $5 \% \mathrm{CO}_{2}$. The cells were treated with dexamethasone (20,100, and $500 \mathrm{nM}$ for $48 \mathrm{~h}$; or $500 \mathrm{nM}$ for 12, 24, $48 \mathrm{~h}$ ). MTS assay was conducted to detect the cytotoxicity of dexamethasone following the manufacturer's protocol. Absorption intensity was measured at $490 \mathrm{~nm}$ using a microplate reader. With the same treatment, the cortisol concentration in culture medium, mRNA and protein expression of related genes were measured. $\mathrm{H} 295 \mathrm{R}$ cells were treated with $500 \mathrm{nM}$ dexamethasone for $24 \mathrm{~h}$ to detected expression of GR and StAR protein, the binding between GR and SF1, and H3K27ac level of SF1 promoter region. For the knockdown of GR or HDAC5, the cells were transfected with siRNA targeting human GR or HDAC5 at a final concentration of 100 pM using Lipofectamine 3000 (Invitrogen, Carlsbad, CA, USA) according to the 
manufacturer's instructions. The siRNA sequences of GR were F: 5'-GGAGAUGACAACUUGACUUTT-3', R: 5'AAGUCAAGUUGUCAUCUCCTT-3'; the siRNA sequences of HDAC5 were F: 5'-GCUAUGACAACGGGAACUUTT3', R: 5'-AAGUUCCCGUUGUCAUAGCTT-3'. After a $24 \mathrm{~h}$ treatment, the cells were harvested for subsequent treatment and analysis.

\subsection{Western blotting analysis}

Homogenate tissues or cells were rinsed with ice-cold PBS and then lysed for 30 minutes at $4^{\circ} \mathrm{C}$ in RIPA lysis buffer containing phosphatase inhibitor cocktail, followed by the BCA Assay Kit for protein quantification. Aliquots of lysate were mixed with $5 \times$ loading buffer containing 2-mercaptoethanol and boiled at $100^{\circ} \mathrm{C}$ for $5 \mathrm{~min}$. Protein samples were separated on $12 \%$ sodium dodecyl sulfate-polyacrylamide (SDS-PAGE) gels before transferring to polyvinylidenedifluoride (PVDF) membranes. Membranes were blocked with $5 \%$ non-fat milk powder for $1 \mathrm{~h}$, and then incubated with various antibodies: GR (1:1500), HDAC5 (1:500), SF1 (1:1000), StAR (1:1000), or GAPDH (1:1000) respectively. After washing with TBST three times, the membranes were incubated with horseradish peroxidase (HRP) conjugated secondary antibodies (1:10000). The protein bands were visualized by using the ECL Plus Kit. Signals of antibody binding were detected by Chemi-doc Image Analyzer (Bio-Rad, Hercules, California). Relative protein level was normalized to GAPDH values and compared to controls.

\subsection{Immunofluorescence analysis}

Following $48 \mathrm{~h}$ treatment as described above, cells were washed with ice-cold PBS for three times, fixed in $4 \%$ formaldehyde, and blocked for 30 min with $3 \%$ of bovine serum albumin (BSA) and $2 \%$ of fetal bovine serum in $0.2 \%$ Triton $\mathrm{X}-100 / \mathrm{PBS}$. The cells were then incubated overnight at $4^{\circ} \mathrm{C}$ with primary antibodies in blocking buffer, include mouse anti-HDAC5 (1:1000 dilutions) and rabbit anti-GR (1:500 dilutions). The cells were washed with PBS and incubated with 1:100 diluted 1:100 diluted FITC-conjugated secondary antibody (GB22301, Servicebio Inc., Wuhan, China) corresponding to anti-GR or anti-HDAC5 for 60 min at room temperature. Nuclei were stained with 4,6-diamidino-2-phenylindole (DAPI) at 1:500 dilution for 5 $\min$. The slides were washed twice with PBS. Negative controls obtained by omitting primary antibody showed negligible background fluorescence. Fluorescence images for GR and HDAC5 were captured using an immunofluorescence microscopy (Nikon H550S, Tokyo, Japan).

\subsection{Co-immunoprecipitation (Co-IP)}

Co-IP of proteins was performed with Protein G Magnetic Beads (Millipore, 16-157) following the manufacturer's instructions. After several washes using ice-cold PBS, $1 \mathrm{ml}$ precooled lysis buffer was added into the cell culture flask for $30 \mathrm{~min}$. The suspension was then centrifuged at $12,000 \mathrm{rpm}, 4^{\circ} \mathrm{C}$ for 15 $\min$. After that, $50 \mu \mathrm{l}$ of the supernatant was used as input protein. The other was divided into $400 \mu \mathrm{l}$ per Eppendorf tube and added with $1 \mu \mathrm{g}$ GR or HDAC5 antibody or a normal IgG of the same species as the negative control and incubated overnight at $4^{\circ} \mathrm{C}$. After that, $40 \mu \mathrm{l}$ of Protein $\mathrm{G}$ Magnetic Beads was added into the above mixture and incubated for $1 \mathrm{~h}$ at $4^{\circ} \mathrm{C}$. The beads-antibody-antigen complexes were collected by centrifugation and washed with lysis buffer for three times. And then, $30 \mu$ loading buffer was added to each sample following with water bath at $100^{\circ} \mathrm{C}$. Western blotting analysis was used to detect the protein level. 


\subsection{Chromatin immunoprecipitation-polymerase chain reaction (ChIP-PCR)}

ChIP assays were performed on adrenal tissues and scraped cells to evaluate the levels of GR-SF1 binding and acetylation of histone 3 Lysine 9, 14 and 27 (H3K9ac, H3K14ac and H3K27ac) at promoter region of SF1 (rat and human) gene according to a modified version of the manufacturer's protocol. The samples were cross-linked with $1 \%$ formaldehyde on a rocker for 10 min and added $125 \mathrm{mM}$ glycine to stop the reaction. The samples were then centrifuged and resuspended in $0.5 \mathrm{ml}$ lysis buffer containing protease inhibitors. Cell lysates were sonicated to shear DNA to lengths of approximately 200 base pairs and transferred to a new tube with ChIP dilution buffer. Chromatin was incubated overnight at $4^{\circ} \mathrm{C}$ on nutator/rocker with specific antibody (1:50 dilution) for GR, H3K9ac, H3K14ac, H3K27ac, IgG and BSAtreated Protein $\mathrm{G}$ beads to reduce nonspecific background binding. The immunoprecipitated DNA-protein complex with beads was collected by centrifugation and washed sequentially with low-salt, high-salt, LiCl immune complex, and Tris-EDTA washing buffer solutions. Freshly prepared elution buffer (1\% SDS, $0.1 \mathrm{M}$ $\mathrm{NaHCO}_{3}$ ) was used to elute the DNA protein complex. The samples were then placed in $65^{\circ} \mathrm{C}$ water baths overnight to reverse formaldehyde cross-linking and subsequently were purified using DNA purification kits. Gene enrichment was quantified relative to input controls by RT-qPCR using primers specific for the promoter region ${ }^{[23]}$. The primers used are shown in Supplemental Table 2.

\subsection{Human subjects, blood collection and PBMC isolation}

This study was approved by the Research Committee for Human Subjects, Zhongnan Hospital of Wuhan University, China (No: 2018050). A retrospective study was conducted with medical records of pregnant women and their neonates from June 1 to December 1, 2017. The following criteria were required for the inclusion: $₫$ neonates who need admission to the neonatal intensive care unit; $₫$ pregnancies who were received one course of dexamethasone treatment between gestation week (GW) 34 to GW42 and delivered after $24 \mathrm{~h}$ to 7 days; or $\otimes$ received one course of dexamethasone treatment at GW23 to GW 33 and delivered after $24 \mathrm{~h}$ to 7 days; $\otimes$ pregnancies who delivered between GW23 to GW42 without administration of dexamethasone. Sixty male neonates recruited in this study were categorized into two groups: control group without dexamethasone administration and dexamethasone group with single dose of treatment. Demographic and pregnancy characteristics of recruited subjects were collected, the detail information is shown in Table 3. We compared maternal age, gestational age, birth weight of neonates and Apgar score of $1 \mathrm{~min}$ and of $5 \mathrm{~min}$ between two groups. Apgar score can describe the condition of the newborn infant and, when properly applied, as a tool to predict infants' outcome. To avoid disturbance of the diseases and multiple pregnancies to the results, pregnant women with abnormal liver function or hereditary metabolic diseases were excluded. All of the donors or their guardians provided written consent and ethics permission was obtained for the use of all samples. Blood samples $(2-3 \mathrm{~mL})$ were obtained with ethylenediaminetetraacetic acid (EDTA) vacutainers within $6 \mathrm{~h}$ after birth, and PBMC were immediately isolated via Ficoll-Paque (catalog no. 17-1440-02, GE) separation. All analyses described below were run in individual samples. 
Table 3

Characteristics of enrolled human subjects.

\begin{tabular}{|llll|}
\hline Indexes & Control $(\mathbf{n}=\mathbf{3 8})$ & ADT $(\mathbf{n}=\mathbf{2 2})$ & P value \\
\hline Maternal age (year) & $31.74 \pm 0.86(22-47)$ & $32.55 \pm 0.10(25-43)$ & 0.533 \\
\hline Gestational age (week) & $34.40 \pm 0.27(31-36.71)$ & $34.03 \pm 0.34(31.29-36.43)$ & 0.399. \\
\hline Birth weight (g) & $2208.68 \pm 62(1500-2910)$ & $2120 \pm 111(960-3250)$ & 0.453. \\
\hline Apgar scores (1 min) & $7.76 \pm 0.12(6-9)$ & $7.68 \pm 0.17(6-9)$ & 0.692. \\
\hline Apgar scores (5 min) & $8.79 \pm 0.12(7-10)$ & $8.77 \pm 0.16(7-10)$ & 0.993 \\
\hline
\end{tabular}

ADT, antenatal dexamethasone treatment. ${ }^{a}$ Values are presented as median (interquartile range); N.S.: No significance.

\subsection{Statistical analysis}

Prism 6.0 (GraphPad Software, La Jolla, CA, USA) was used to perform data analysis. All presented measurement data were expressed as the mean \pm S.E.M. and was evaluated with Independent Samples $t$ test for comparison between two groups or with one-way ANOVA followed by a post hoc Dunnett $t$-test for comparison among multiple groups. Statistical significance was set at $P<0.05$.

\section{Result}

\subsection{Changes of adrenal morphology and steroid synthesis function in male fetal rats induced by PDE}

This study adopted the rat PDE model (dexamethasone 0.2 and $0.8 \mathrm{mg} / \mathrm{kg} . \mathrm{d}$ s.c., GD9-20) ${ }^{[24]}$ to observe the changes of adrenal morphology and steroid synthesis function in male fetal rats. HE staining results confirmed that the maximum cross-sectional area and diameter of adrenal in PDE group were significantly lower than those in control group $(P<0.05$, Fig. 1A,1B), which suggested fetal adrenal morphological dysplasia. Subsequently, we analyzed the gene expression profile of male fetal adrenal for understanding the change of fetal adrenal function. The heat map results showed that there is a good separation between PDE and control groups (Fig. 1C). GO analysis indicated that the up-regulated pathways in PDE group mainly included: complement and coagulation cascades, serotonergic synapse and so on (Fig. 1D); the down-regulated pathways in PDE group mainly include: glycerophospholipid metabolism, valine, leucine and isoleucine degradation and so on (Fig. 1E). Furthermore, we discovered that the changes of fetal serum corticosterone concentration, adrenal steroid synthesis system and SF1 mRNA expression in PDE group were significantly lower than control group $(P<0.05, P<0.01$, Fig. 1F,1G). Meanwhile, we confirmed that protein expression of SF1 and StAR in PDE group were decreased $(P<0.05, P<0.01$, Fig. $1 \mathrm{H})$. In conclusion, PDE caused fetal adrenal dysplasia and steroid synthesis function inhibition in male fetal rats. 


\subsection{Changes of adrenal morphology and steroid synthesis function after birth in male offspring rats induced by PDE}

Next, we observed the changes of adrenal morphology and steroid synthesis function in PDE male offspring rats after birth. The results showed that adrenal maximum cross-sectional area and diameter of PDE male offspring were significantly lower than those of control group $(P<0.05, P<0.01$, Fig. 2A-2C), and serum corticosterone level was significantly decreased $(P<0.05, P<0.01$, Fig. 2D). Meanwhile, SF1, StAR, P450scc and P450c21 mRNA expression in PW6; SF1, StAR and P450scc mRNA expression in PW8; and SF1, StAR and P450c11 mRNA expression in PW28 were decreased in PDE group compared with control group $(P<0.05, P<0.01$, Fig. 2E-2G). However, there was no significant change in other indicators at above time points (Fig. 2E-2G). Furthermore, the protein expression of SF1 and StAR were also decreased in PDE group after birth $(P<0.05, P<0.01$, Fig. $2 \mathrm{H}-2 \mathrm{~L})$. Therefore, PDE led to adrenal dysplasia and decreased steroid synthesis function in male offspring after birth, especially for SF1 and StAR expression.

\subsection{Changes of GR/HDAC5 expression and epigenetic modification of SF1/StAR in male offspring rats before and after birth induced by PDE}

To explore the epigenetic mechanism in low expression programming of SF1 and StAR, we detected the histone acetylation level in adrenal SF1 and StAR promoter region of male offspring rats. In SF1 promoter region of PDE group, H3K9ac, H3K14ac and H3K27ac levels in GD20, H3K14ac and H3K27ac levels in PW6, H3K9ac and H3K27ac levels in PW8, and H3K9ac and H3K27ac levels in PW28 were all decreased, but other indicators showed no significant changes $(P<0.05, P<0.01$, Fig. 3A-3D), suggesting that H3K27ac level in SF1 promoter region could be reduced from intrauterine to postnatal. Meanwhile, in StAR promoter region of PDE group, H3K9ac and H3K27ac levels in GD20, H3K14ac and H3K27ac levels in PW6, H3K14ac, H3K27ac levels in PW8, and H3K9ac level in PW28 were decreased $(P<0.05, P<0.01$, Fig. 3E$3 \mathrm{H})$, suggesting that $\mathrm{H} 3 \mathrm{~K} 27 \mathrm{ac}$ levels in StAR promoter region could also be reduced from intrauterine to postnatal except in PW28. Therefore, H3K27ac in SF1 promoter region might be an epigenetic biomarker for adrenal insufficiency in male PDE rats.

To explore the occurrence mechanism of persistent decreased H3K27ac level of SF1 promoter region in PDE offspring rats, we screened differential expression HDACs involved in regulating adrenal histone acetylation in male fetal rats, and found that only HDAC5 expression was increased (Fig. 3I). Subsequently, we confirmed that the mRNA and protein levels of GR and HDAC5 were increased in PDE group $(P<0.05, P$ $<0.01$, Fig. 3J-3K). Therefore, PDE can increase adrenal GR/HDAC5 signal in male fetal rats.

\subsection{Changes of dexamethasone on HDAC5/SF1/StAR expression and cortisol production in human adrenocortical cells}


Our previous study found that concentration of dexamethasone in male fetal serum in $\operatorname{PDE}(\mathrm{L})$ group was $0.105 \mu \mathrm{g} / \mathrm{mL}(267 \mathrm{nM})^{7}$. Therefore, we observed the effects of dexamethasone on HDAC5/SF1/StAR expression and cortisol production in H295R cells. Firstly, MTS experiment showed that different concentrations of dexamethasone $(0,20,100,500 \mathrm{nM})$ had no significant effect on cell viability in $48 \mathrm{~h}$ (Fig. 4A). Then, we treated the cells with $500 \mathrm{nM}$ dexamethasone at different time $(0,12,24,48 \mathrm{~h})$ or dexamethasone at different concentrations $(0,20,100,500 \mathrm{nM})$ for $48 \mathrm{~h}$, respectively. The results showed that dexamethasone decreased the culture medium cortisol level in concentration- and time-dependence ( $P$ $<0.05, P<0.01$, Fig. 4B, 4C). Meanwhile, dexamethasone increased the mRNA and protein expression levels of HDAC5 and inhibited those of SF1 and StAR $(P<0.05, P<0.01$, Fig. 4D-4G). Therefore, dexamethasone could increase the expression of HDAC5, inhibit the expression of SF1 and StAR, and reduce cortisol production.

\subsection{GR/SF1-mediated the effects of dexamethasone on SF1 expression and steroid synthesis function in human adrenocortical cells}

We further investigated the regulatory mechanism that dexamethasone down-regulated adrenal SF1 expression and inhibited steroid synthesis. Firstly, we treated the cells with dexamethasone at different concentrations $(0,20,100,500 \mathrm{nM})$ for $48 \mathrm{~h}$, and found that dexamethasone could increase GR mRNA expression $(P<0.05, P<0.01$, Fig. 5A). Meanwhile, we extracted of cytosol and nuclear protein after treatment with $500 \mathrm{nM}$ dexamethasone, and found that dexamethasone decreased GR expression in cytoplasm but increased GR expression in nucleus compared with their control groups (Fig. 5B). These results indicated that dexamethasone could activate $\mathrm{GR}$. We further confirmed that GR siRNA could reduce the expression of GR mRNA ( $P<0.01$, Fig. $5 C$ ), suggesting that GR silence was successful. Then, we treated the cells with $500 \mathrm{nM}$ dexamethasone alone or in combination with GR siRNA. The results showed that dexamethasone alone could inhibit the mRNA and protein expression of SF1 and StAR, and reduce the cortisol level in the culture medium, while GR siRNA could reverse the effect of dexamethasone $(P<0.05, P$ $<0.01$, Fig. 5D-5I). Finally, we predicted the binding sites between GR and SF1 promoter region by bioinformatics (Fig. 5J), and confirmed that dexamethasone could increase the binding sites of GR and SF1 promoter regions, while GR siRNA could reverse the binding effect $(P<0.05, P<0.01$, Fig. $5 \mathrm{~J})$. In conclusion, dexamethasone down-regulated the mRNA and protein expression of SF1 by activating GR and binding to SF1 promoter region, and then inhibit StAR expression and cortisol production.

\subsection{GR/HDAC5 signal-mediated epigenetic regulating mechanism of dexamethasone on SF1 expression inhibition in human adrenocortical cells}

We also investigated the epigenetic mechanism that HDAC5 mediated the decreased SF1 expression of adrenocortical cells induced by dexamethasone. Firstly, we treated the H295R cells with $500 \mathrm{nM}$ 
dexamethasone alone or in combination HDAC5 siRNA. HDAC5 siRNA could decrease mRNA expression of HDAC5 $(P<0.01$, Fig. 6A), suggested that HDAC5 silence was successful. Dexamethasone could increase the mRNA expression of HDAC5 $(P<0.01$, Fig. 6A), and decrease H3K27ac level of SF1 promoter region, the mRNA and protein expression of SF1 and StAR and cortisol level $(P<0.01$, Fig. 6B-6H), while HDAC5 siRNA reversed the above effects of dexamethasone $(P<0.05$, Fig. 6B-6H); furthermore, GR siRNA could reversed the above effects of dexamethasone $(P<0.05, P<0.01$, Fig. $6 \mathrm{I}-6 \mathrm{~K})$. We also confirmed the colocalization of GR and HDAC5 in H295R cells (Fig. 6L). Finally, we confirmed that dexamethasone could increase the combination between GR and HDAC5 (Fig. 6M). In conclusion, GR up-regulated and recruited HDAC5, reducing H3K27ac level of SF1 promoter region and its expression, and inhibiting the expression of StAR and cortisol production.

\subsection{Correlation analysis between H3K27ac level of SF1 promoter region and its expression in animal and population experiments}

We detected H3K27ac level of SF1 promoter region in PBMC of PDE male rats and its expression for an early warning target of peripheral blood for adrenal insufficiency. The results showed that H3K27ac levels of SF1 promoter region and its expression in PBMC of male PDE offspring rats were decreased compared with control group in GD20, PW6, PW8 and PW28 $(P<0.01$, Fig. 7A-7H). Furthermore, we analyzed the correlations between adrenal tissue and PBMC in H3K27ac level of SF1 promoter region and its expression. The results showed that there were positive correlations between adrenal tissue and PBMC in H3K27ac level of SF1 promoter region and its expression before and after birth $(P<0.05, P<0.01$, Fig. 7I7P) in male PDE group, but not in control group (Fig. 7I-7P). Therefore, H3K27ac level of SF1 promoter region in PBMC of male PDE offspring rats could reflect the related changes in adrenal tissue.

For verifying clinic peripheral blood epigenetic markers of adrenal insufficiency in male offspring (newborns) of prenatal dexamethasone application, we collected male neonatal plasma samples from applying one course of dexamethasone during pregnancy and matched gestational age control. Firstly, we performed a statistical analysis of clinically relevant information, and confirmed that there were no significant differences in maternal age, gestational age, birth weight and Apgar score at birth between control and dexamethasone groups (Supplemental Table 3). Moreover, we extracted neonatal PBMC, detected H3K27ac level of SF1 promoter region and its expression, and analyzed its correlation. The results showed that H3K27ac levels of SF1 promoter region and their expression were significantly decreased in neonatal PBMC of prenatal dexamethasone application compared with control $(P<0.05$, Fig. 7R-7S), and there were positive correlations between H3K27ac level of SF1 promoter region and its expression in dexamethasone group, but not in control group $(P<0.01$, Fig. 7T). Therefore, prenatal dexamethasone application could reduce H3K27ac level of SF1 promoter region and its expression in neonatal PBMC.

\section{Discussion}




\subsection{The rationality of dose and time, and persistent adrenal insufficiency in PDE male offspring rats}

Synthetic glucocorticoids are used for pregnant women with premature birth tendency once a week [25]. However, single treatment for some pregnant women was not significant for the difficulty in early diagnosis of preterm birth, therefore, about one third of them changed from preventive medication to continuous treatment ${ }^{[26]}$, some of them would accept multiple courses of treatment ${ }^{[27]}$. $0.05-0.2 \mathrm{mg} / \mathrm{kg} \cdot \mathrm{d}$ was proved to be close to human treatment dose in studying long-term effect of synthetic glucocorticoids in pregnant rats ${ }^{[28]}$. In this study, the doses of PDE were 0.2 and $0.8 \mathrm{mg} / \mathrm{kg} \cdot \mathrm{d}$, and only low-dose group was retained after birth (the offspring rat mortality rate after birth was higher in high dose group). Combining with dose conversion relationship between rats and humans in drug administration (conversion coefficient is 6.17), the rat dose of PDE in this study was equal to 0.03 and $0.12 \mathrm{mg} / \mathrm{kg} \cdot \mathrm{d}$ in human, so the dose of dexamethasone in this study met clinical criteria. In addition, dexamethasone was used to treat pregnant women at risk for premature delivery at 24-34 weeks (middle and late pregnancy) ${ }^{[29]}$, while rat adrenocortical differentiation began from GD9 (the second trimester) ${ }^{[30]}$. Therefore, we chose a rat model (GD9-20, 0.2 and $0.8 \mathrm{mg} / \mathrm{kg} \cdot \mathrm{d}$ ) to explore the effect of PDE on rats adrenal steroid synthesis function.

Glucocorticoids is an key metabolic regulator, which could promote fetal development and multi-organ maturation. However, fetal development is easily affected by internal and external environmental stimuli. Prenatal maternal glucocorticoids overexposure inhibited fetal adrenal development and steroid hormone synthesis ${ }^{[31-32]}$. This study discovered that PDE inhibited the development of adrenal morphology and function in male fetal rats, which was mainly reflected in significant decrease of the maximum crosssectional area/diameter of adrenal and serum CORT level, which continued after birth. Glucocorticoids could regulate the biosynthesis and metabolism of sugar, fat and protein, and involve in the occurrence of many chronic diseases. As synthetic organ of glucocorticoids, adrenal is continuous low function, which leads to the decrease of circulating glucocorticoid level and metabolic disorder of body and mediates the occurrence of fetal-originated diseases.

\subsection{Persistent adrenal insufficiency of steroid hormone synthesis in male offspring induced by PDE associated with the inhibition of SF1/StAR expression caused by dexamethasone}

Glucocorticoids synthesis is catalyzed by multiple steroid synthase systems, StAR transporting cholesterol is the first step ${ }^{[9]}$. The gene promoter region of the above steroid synthases exist the identification sequence of SF1 ${ }^{[33,34]}$, which are regulated by SF1. Our previous research discovered that deoxycorticosterone (an intermediate product of corticosterone synthesis) was reduced by fetal serum metabolic spectrum of PDE rats, suggesting that PDE could inhibit fetal adrenal function ${ }^{[35]}$. In this study, we found that gene and protein expression of steroid hormone synthesis system and their transcription 
factors SF1 were inhibited before birth (especially for SF1 and StAR). Further, we confirmed that dexamethasone could significantly inhibit SF1 gene and protein expression in human adrenocortical cells. Therefore, low adrenal function caused by PDE in male offspring rats may be related to the inhibition of SF1/StAR expression.

\subsection{Dexamethasone inhibits H3K27ac level of SF1 promoter region and its expression by GR/HDAC5 double pathway in adrenal}

"Intrauterine programming" is associated with epigenetic modifications mediated by aberrant programming of multiorgan development ${ }^{[36,37]}$. Our previous studies have found that PDE can induce the abnormal modification of histone acetylation of key genes, which mediates the occurrence of osteoporosis and testicular dysplasia ${ }^{[24,38]}$. In this study, we found that only reduced H3K27ac level of SF1 promoter region in PDE group lasted from intrauterine to post-natal. Therefore, low expression of SF1 caused by low $\mathrm{H} 3 \mathrm{~K} 27 \mathrm{ac}$ level in SF1 promoter region mediated the reduced function programming of adrenal in male rats caused by PDE, while decreased expression of StAR resulted from transcriptional regulation of SF1.

We further explored the molecular mechanism of reduced H3K27ac level of SF1 promoter region induced by dexamethasone. We discovered that dexamethasone decreased the expression of SF1/StAR and cortisol level by up-regulating and activating GR in H295R cell. GR siRNA could reverse the effect induced by dexamethasone. Then, we confirmed that dexamethasone could increase the binding between GR and HDAC5, while GR siRNA could reverse the effect. These results suggested that dexamethasone downregulated SF1 expression by activating GR and combining with SF1 promoter region, and then inhibited StAR expression and cortisol production.

We explored the molecular mechanism of decreased H3K27ac level in SF1 promoter region induced by dexamethasone. GR binds with glucocorticoids and activated into nucleus, combining with glucocorticoids response element in target gene, and HDACs could be recruited to regulate jointly gene transcription [39]. Histone deacetylation is a common form of epigenetic modification, and high HDACs expression is accompanied by high level of histone deacetylation, thus affecting gene expression ${ }^{[40]}$. Our results in vitro showed that PDE could increase the expression of GR and HDAC5 in fetal adrenal of male rats. In vivo, dexamethasone could increase HDAC5 expression, and reduced H3K27ac level of SF1 promoter region, the expression of SF1/StAR and cortisol level of culture medium. HDAC5 siRNA could reverse the effect. We also confirmed the co-localization of GR and HDAC5 in H295R cells. Finally, we confirmed that dexamethasone enhanced the interaction between GR and HDAC5. The results indicated that GR upregulated and recruited HDAC5, decreased H3K27ac level of SF1 promoter region and inhibited SF1 expression, and then inhibited StAR expression and cortisol production. 


\subsection{The H3K27ac level of SF1 promoter region in PBMC may be used as a biomarker of PDE adrenal insufficiency}

Biomarkers can provide guidance for early diagnosis and treatment of diseases ${ }^{[40,42]}$. The related gene expression and epigenetic modification of peripheral blood in disease conditions may reflect the consistency of gene expression and epigenetic modifications of target organs $[43,44]$, and the similar changes in blood could be used as biomarker of disease phenotype ${ }^{[45]}$. In disease state, the expression of PBMC gene in patients was consistent with gene expression in animal tissues of disease model, such as the gene expression of survivin in obesity ${ }^{[46]}$. The abnormal epigenetic modifications of related genes could be used as biomarker of disease in disease state, such as the acetylated modification change of peripheral blood leukocyte endothelin 1 could be used as the biomarkers for pulmonary vascular disease of intrauterine retarded offspring or subsequent asthma ${ }^{[47]}$. In this study, we found that H3K27ac level of SF1 promoter region and its expression in PDE male progeny rat PBMC were decreased, showing a positive correlation with the same index in adrenal tissue. It was very meaningful that H3K27ac level of SF1 promoter region and its expression in dexamethasone group were also decreased in clinical trial, and there was a positive correlation between them. These results suggested that decreased H3K27ac level of SF1 promoter region and its expression in PBMC may be the biomarkers of adrenal insufficiency in male offspring caused by PDE in clinic.

\section{Conclusion}

To sum up, activating GR by dexamethasone, on one hand, combining with SF1 promoter region and inhibiting its expression, on the other hand, up-regulating and recruiting HDAC5 and decreasing H3K27ac level of SF1 promoter region, then inhibiting SF1 expression together (Fig. 8). The epigenetic modification played a major role in this process, decreased H3K27ac level of adrenal SF1 promoter region in PDE offspring rats was programmed and continued after birth. In addition, level of H3K27ac in adrenal SF1 promoter region of PDE offspring rats was significantly positively correlated with level of H3K27ac in SF1 promoter region of PBMC, and $\mathrm{H} 3 \mathrm{~K} 27 \mathrm{ac}$ in SF1 promoter region and its expression were also significantly decreased in neonates treated by dexamethasone. These results suggested that low H3K27ac of PBMC mediated persistent adrenal dysfunction in PDE offspring rats and it could be used as an early warning marker of adrenal low function (Fig. 8).

\section{Declarations}

\section{Funding}

This work was supported by grants from the National Natural Science Foundation of China (No. 81673524), the National Key Research and Development Program of China (No. 2017YFC1001300), the Major Technological Innovation Projects of Hubei Province (No囚2019ACA140), and Medical Science Advancement Program (Basic Medical Sciences) of Wuhan University, Grant No. TFJC2018001. 
The authors declare no competing interests.

Ethics approval

Compliance with ethical standards. The experimental protocol was approved by the Ethics Committee for Animal (No:14016) and Human (No: 2018050) Experimentsof the Medical College of Wuhan University.

Consent to participate

Not applicable.

Consent for publication

Not applicable.

Availability of data and material

All data generated or analyzed during this study are included in this published article.

Code availability

Not applicable.

Authors' contributions

Guanghui Chen, Can Ai and Jiangang Cao performed the research, Hui Wang designed the research study, Fangfang duan, Jinzhi Zhang and Yawen Chen analyzed the data, Guanghui Chen, Ying Ao and Hui Wang wrote and revised the paper, all authors approved the final manuscript.

\section{References}

1. Chawanpaiboon S, Vogel JP, Moller AB, Lumbiganon P, Petzold M, Hogan D, et al. Global, regional, and national estimates of levels of preterm birth in 2014: a systematic review and modelling analysis. The Lancet Global health. 2019;7(1):e37-46.

2. Roberts D, Brown J, Medley N, Dalziel SR. Antenatal corticosteroids for accelerating fetal lung maturation for women at risk of preterm birth. The Cochrane database of systematic reviews 2017, 3(3): Cd004454.

3. Onland W, De Jaegere AP, Offringa M, van Kaam A. Systemic corticosteroid regimens for prevention of bronchopulmonary dysplasia in preterm infants. The Cochrane database of systematic reviews 2017, 1(1): Cd010941.

4. Vogel JP, Souza JP, Gülmezoglu AM, Mori R, Lumbiganon P, Qureshi Z, et al. Use of antenatal corticosteroids and tocolytic drugs in preterm births in 29 countries: an analysis of the WHO Multicountry Survey on Maternal and Newborn Health. Lancet. 2014;384(9957):1869-77. 
5. Asztalos EV, Murphy KE, Hannah ME, Willan AR, Matthews SG, Ohlsson A, et al. Multiple courses of antenatal corticosteroids for preterm birth study: 2-year outcomes. Pediatrics. 2010;126(5):e1045-55.

6. Elfayomy AK, Almasry SM. Effects of a single course versus repeated courses of antenatal corticosteroids on fetal growth, placental morphometry and the differential regulation of vascular endothelial growth factor. J Obstet Gynaecol Res. 2014;40(11):2135-45.

7. Xu D, Chen M, Pan XL, Xia LP, Wang H. Dexamethasone induces fetal developmental toxicity through affecting the placental glucocorticoid barrier and depressing fetal adrenal function. Environmental toxicology pharmacology. 2011;32(3):356-63.

8. Quinn TA, Ratnayake U, Castillo-Melendez M, Moritz KM, Dickinson H, Walker DW. Adrenal steroidogenesis following prenatal dexamethasone exposure in the spiny mouse. J Endocrinol. 2014;221(2):347-62.

9. Jefcoate CR, Lee J. Cholesterol signaling in single cells: lessons from STAR and sm-FISH. J Mol Endocrinol. 2018;60(4):R213-r235.

10. Gardiner JR, Shima Y, Morohashi K, Swain A. SF-1 expression during adrenal development and tumourigenesis. Molecular cellular endocrinology. 2012;351(1):12-8.

11. Morohashi K, Honda S, Inomata Y, Handa H, Omura T. A common trans-acting factor, Ad4-binding protein, to the promoters of steroidogenic P-450s. J Biol Chem. 1992;267(25):17913-9.

12. Moisiadis VG, Matthews SG. Glucocorticoids and fetal programming part 2: Mechanisms. Nature reviews Endocrinology. 2014;10(7):403-11.

13. Heard E, Martienssen RA. Transgenerational epigenetic inheritance: myths and mechanisms. Cell. 2014;157(1):95-109.

14. Liu L, Wang JF, Fan J, Rao YS, Liu F, Yan YE, et al. Nicotine Suppressed Fetal Adrenal StAR Expression via YY1 Mediated-Histone Deacetylation Modification Mechanism. International journal of molecular sciences 2016, 17(9).

15. Yan YE, Liu L, Wang JF, Liu F, Li XH, Qin HQ, et al. Prenatal nicotinic exposure suppresses fetal adrenal steroidogenesis via steroidogenic factor 1 (SF-1) deacetylation. Toxicol Appl Pharmcol. 2014;277(3):231-41.

16. Xu D, Wu Y, Liu F, Liu YS, Shen L, Lei YY, et al. A hypothalamic-pituitary-adrenal axis-associated neuroendocrine metabolic programmed alteration in offspring rats of IUGR induced by prenatal caffeine ingestion. Toxicol Appl Pharmcol. 2012;264(3):395-403.

17. Harris A, Seckl J. Glucocorticoids, prenatal stress and the programming of disease. Hormones behavior. 2011;59(3):279-89.

18. Giussani M, Triulzi T, Sozzi G, Tagliabue E. Tumor Extracellular Matrix Remodeling: New Perspectives as a Circulating Tool in the Diagnosis and Prognosis of Solid Tumors. Cells 2019, 8(2).

19. Narimatsu $H$, Sato T. Wisteria floribunda agglutinin positive glycobiomarkers: a unique lectin as a serum biomarker probe in various diseases. Expert Rev Proteomics. 2018;15(2):183-90.

20. Ubolyam S, lampornsin T, Sophonphan J, Avihingsanon A, Suwanpimolkul G, Kawkitinarong K, et al. Performance of a simple flow cytometric assay in diagnosing active tuberculosis. Tuberc (Edinb 
Scotl). 2021;126:102017.

21. Fougère $B$, Landkocz Y, Lepers $C$, Martin PJ, Armand L, Grossin N, et al. Influence of aging in the modulation of epigenetic biomarkers of carcinogenesis after exposure to air pollution. Experimental gerontology. 2018;110:125-32.

22. Parashar S, Cheishvili D, Mahmood N, Arakelian A, Tanvir I, Khan HA, et al. DNA methylation signatures of breast cancer in peripheral T-cells. BMC Cancer. 2018;18(1):574.

23. Kimura K, Hohjoh H, Fukuoka M, Sato W, Oki S, Tomi C, et al. Circulating exosomes suppress the induction of regulatory T cells via let-7i in multiple sclerosis. Nature communications. 2018;9(1):17.

24. Liu M, Chen B, Pei L, Zhang Q, Zou Y, Xiao H, et al. Decreased H3K9ac level of StAR mediated testicular dysplasia induced by prenatal dexamethasone exposure in male offspring rats. Toxicology. 2018;408:1-10.

25. Antenatal corticosteroids revisited: repeat courses - National Institutes of Health Consensus Development Conference Statement, August 17-18. 2000. Obstetrics and gynecology 2001, 98(1): 144-150.

26. Murphy KE, Hannah ME, Willan AR, Hewson SA, Ohlsson A, Kelly EN, et al. Multiple courses of antenatal corticosteroids for preterm birth (MACS): a randomised controlled trial. Lancet. 2008;372(9656):2143-51.

27. Quinlivan JA, Evans SF, Dunlop SA, Beazley LD, Newnham JP. Use of corticosteroids by Australian obstetricians-a survey of clinical practice. Aust N Z J Obstet Gynaecol. 1998;38(1):1-7.

28. Moisiadis VG, Matthews SG. Glucocorticoids and fetal programming part 1: Outcomes. Nature reviews Endocrinology. 2014;10(7):391-402.

29. Kemp MW, Newnham JP, Challis JG, Jobe AH, Stock SJ. The clinical use of corticosteroids in pregnancy. Hum Reprod Update. 2016;22(2):240-59.

30. Eguchi Y, Ariyuki F. DEVELOPMENT OF THE FETAL RAT. ADRENAL IN PROLONGED PREGNANCY. Endocrinologia japonica 1963, 10: 125-135.

31. He Z, Zhu C, Huang H, Liu L, Wang L, Chen L, et al. Prenatal caffeine exposure-induced adrenal developmental abnormality in male offspring rats and its possible intrauterine programming mechanisms. Toxicology research. 2016;5(2):388-98.

32. Huang H, He Z, Zhu C, Liu L, Kou H, Shen L, et al. Prenatal ethanol exposure-induced adrenal developmental abnormality of male offspring rats and its possible intrauterine programming mechanisms. Toxicol Appl Pharmcol. 2015;288(1):84-94.

33. Hu MC, Hsu NC, Pai Cl, Wang CK, Chung B. Functions of the upstream and proximal steroidogenic factor 1 (SF-1)-binding sites in the CYP11A1 promoter in basal transcription and hormonal response. Molecular endocrinology (Baltimore Md). 2001;15(5):812-8.

34. Ben-Zimra M, Koler M, Orly J. Transcription of cholesterol side-chain cleavage cytochrome P450 in the placenta: activating protein-2 assumes the role of steroidogenic factor- 1 by binding to an overlapping promoter element. Molecular endocrinology (Baltimore Md). 2002;16(8):1864-80. 
35. Chen G, Xiao H, Zhang J, Zhang H, Li B, Jiang T, et al. Prenatal dexamethasone exposure-induced a gender-difference and sustainable multi-organ damage in offspring rats via serum metabolic profile analysis. Toxicology letters. 2019;316:136-46.

36. Charles MA, Delpierre C, Bréant B [Developmental origin of health and adult diseases (DOHaD): evolution of a concept over three decades]. Medecine sciences: M/S 2016, 32(1): 15-20.

37. Godfrey KM, Costello PM, Lillycrop KA. Development, Epigenetics and Metabolic Programming. Nestle Nutrition Institute workshop series. 2016;85:71-80.

38. Xiao H, Wen Y, Pan Z, Shangguan Y, Qin J, Tan Y, et al. Increased H3K27ac level of ACE mediates the intergenerational effect of low peak bone mass induced by prenatal dexamethasone exposure in male offspring rats. Cell death disease. 2018;9(6):638.

39. Kassel O, Herrlich P. Crosstalk between the glucocorticoid receptor and other transcription factors: molecular aspects. Molecular cellular endocrinology. 2007;275(1-2):13-29.

40. Grabiec AM, Potempa J. Epigenetic regulation in bacterial infections: targeting histone deacetylases. Crit Rev Microbiol. 2018;44(3):336-50.

41. Nebbioso A, Tambaro FP, Dell'Aversana C, Altucci L. Cancer epigenetics: Moving forward. 2018, 14(6): e1007362.

42. Lomberk G, Blum Y. Distinct epigenetic landscapes underlie the pathobiology of pancreatic cancer subtypes. 2018, 9(1): 1978.

43. Ma B, Wilker EH, Willis-Owen SA, Byun HM, Wong KC, Motta V, et al. Predicting DNA methylation level across human tissues. Nucleic acids research. 2014;42(6):3515-28.

44. Davies MN, Volta M, Pidsley R, Lunnon K, Dixit A, Lovestone S, et al. Functional annotation of the human brain methylome identifies tissue-specific epigenetic variation across brain and blood. Genome biology. 2012;13(6):R43.

45. Hosseini A, Mirzaei A, Salimi V, Jamshidi K, Babaheidarian P, Fallah S, et al. The local and circulating SOX9 as a potential biomarker for the diagnosis of primary bone cancer. Journal of bone oncology. 2020;23:100300.

46. Kundakovic M, Gudsnuk K, Herbstman JB, Tang D, Perera FP, Champagne FA. DNA methylation of BDNF as a biomarker of early-life adversity. Proc Natl Acad Sci USA. 2015;112(22):6807-13.

47. Xu XF, Xu SS, Fu LC, Hu QY, Lv Y, Du LZ. Epigenetic changes in peripheral leucocytes as biomarkers in intrauterine growth retardation rat. Biomedical reports. 2016;5(5):548-52.

\section{Supplemental}

Supplemental Tables 1-3 are not available with this version

\section{Figures}




\section{A CON}
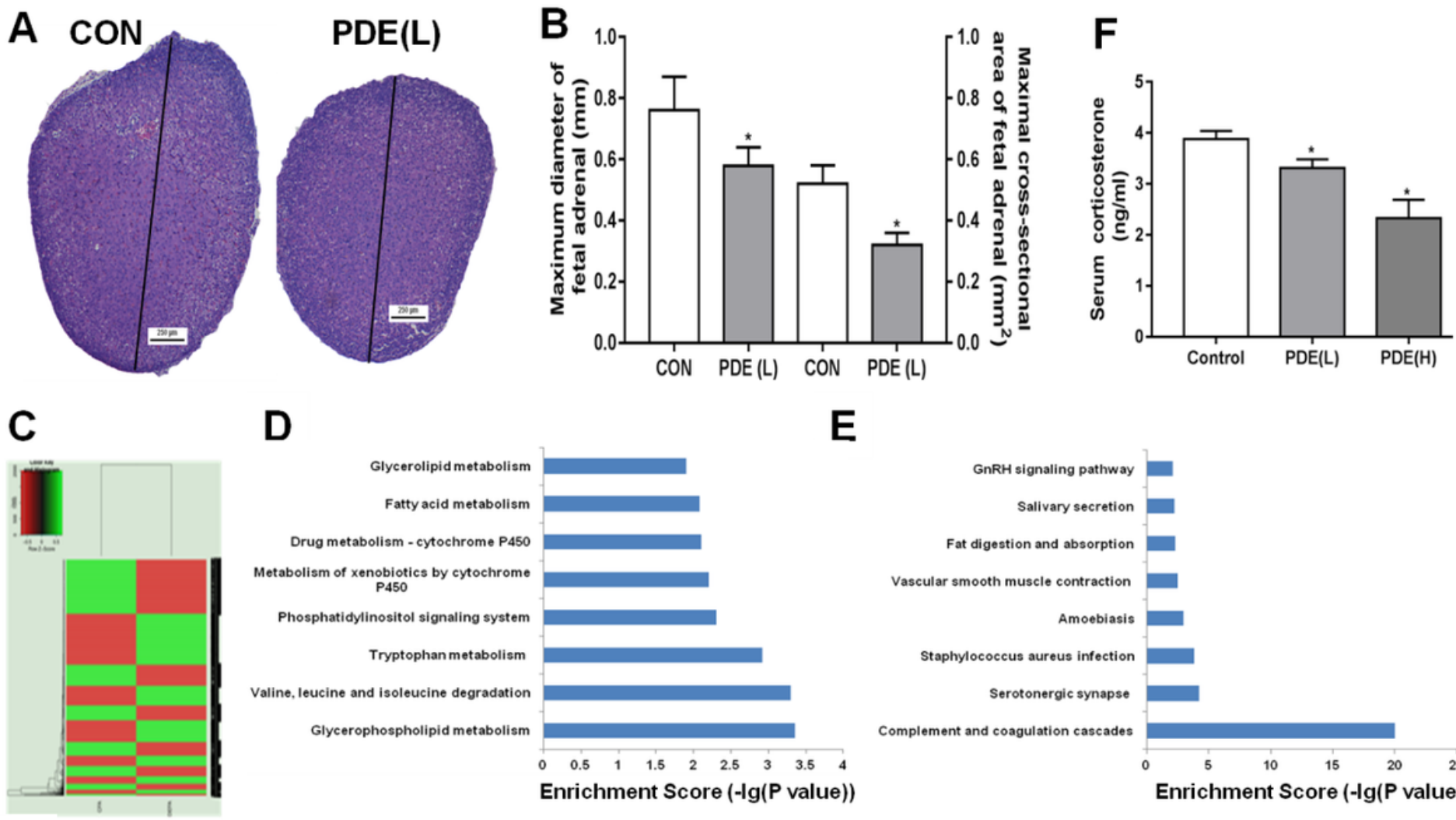

D
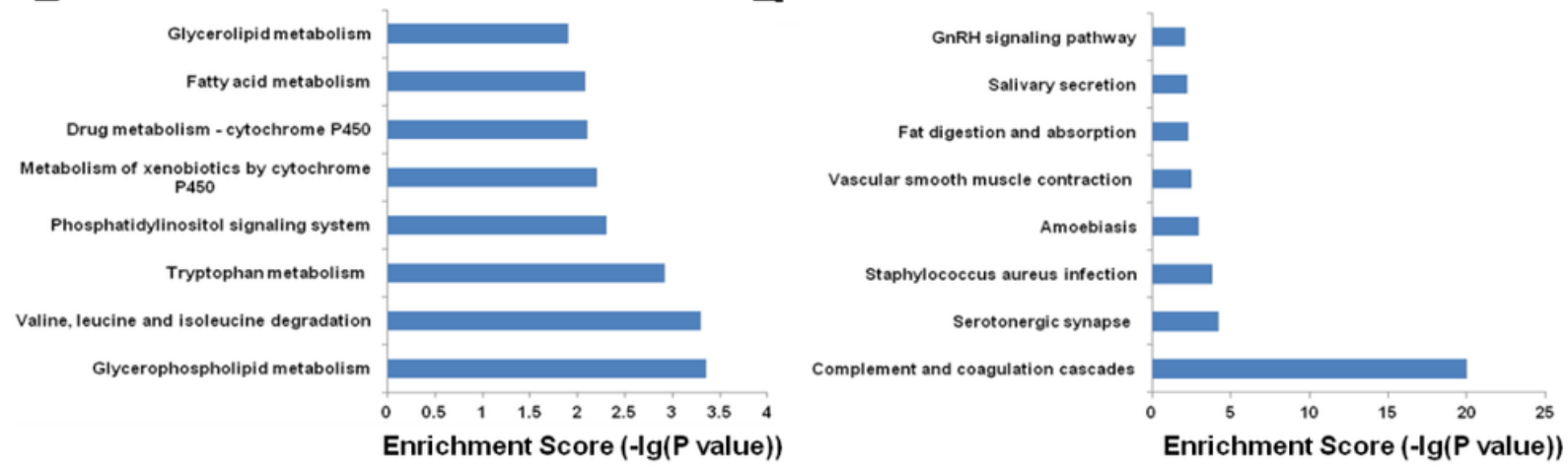

G

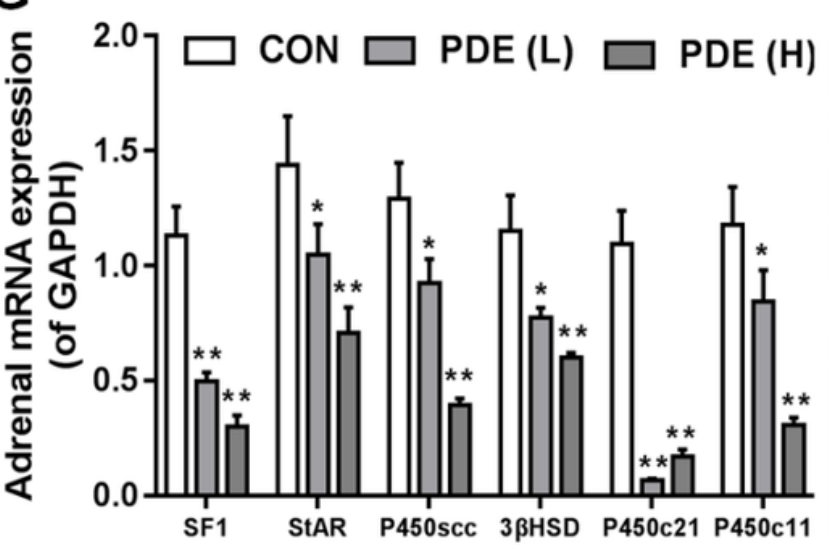

H CON
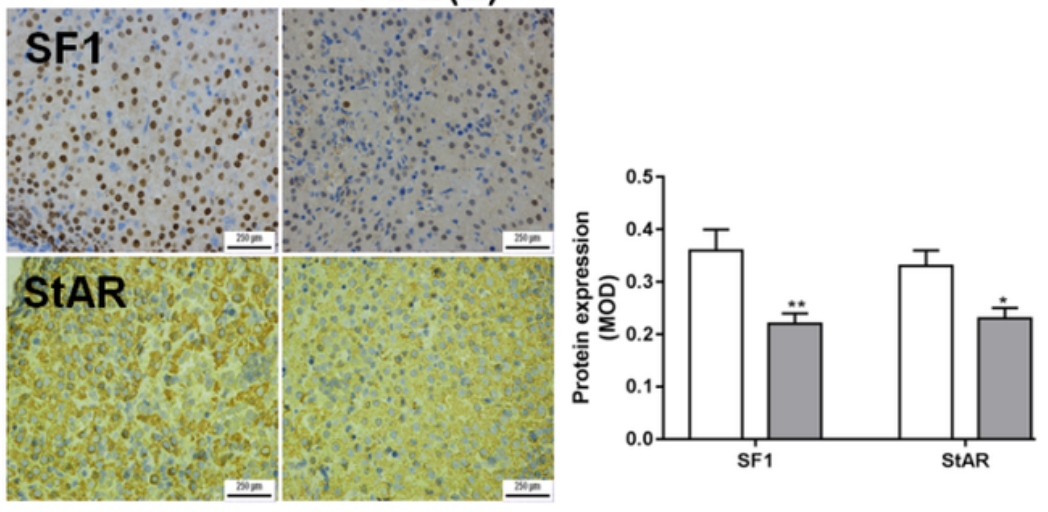

Figure 1

Effects of prenatal dexamethasone exposure (PDE) on adrenal morphology and steroid synthesis function in male fetal rats. A: Adrenal sections were stained with hematoxylin and eosin reagents (magnification: $\times 200$ ), B: Adrenal maximal cross-sectional area and diameter, $n=3$, C: Heat map for micro-array of fetal adrenal gland, D,E: GO analysis for micro-array of fetal adrenal gland, F: Serum corticosterone level was measured by enzyme-linked immunosorbent assay (ELISA), $n=12$, G: mRNA expressions of steroidogenic factor 1 (SF1), steroidogenic acute regulatory protein (StAR), cytochrome P450 cholesterol side-chain

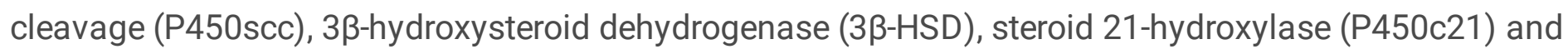
steroid 11 $\beta$-hydroxylase (P450c21) were determined by real-time quantitative polymerase chain reaction (RT-qPCR), $n=12, \mathrm{H}$ : Protein expressions of SF1 and StAR were determined by immumohistochemical staining (magnification: $\times 200$ ), $n=3$ (three fetal adrenals from three littermates were pooled for homogenization into one sample). Mean \pm S.E.M., ${ }^{*} P<0.05,{ }^{*} P<0.01$ vs control. 

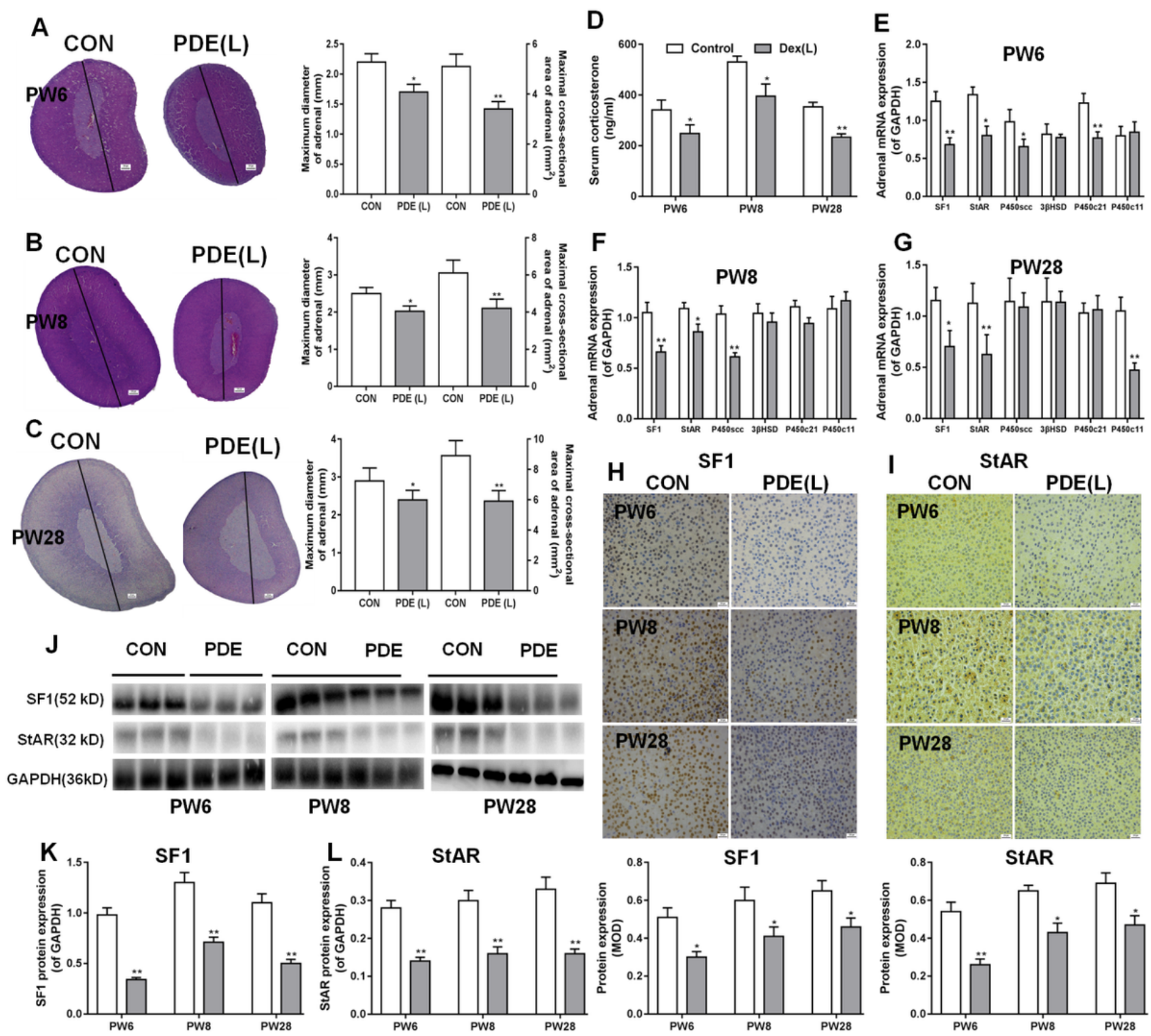

Figure 2

Effects of prenatal dexamethasone exposure (PDE) on adrenal morphology and steroid synthesis function in male offspring rats. A-C: Adrenal maximal cross-sectional area and diameter at postnatal week (PW) 6 , PW8 and PW28 in hematoxylin and eosin reagents (magnification: $\times 200$ ), n=3, D: Serum corticosterone level measured by enzyme-linked immunosorbent assay (ELISA), Mean \pm S.E.M., $n=12, E-G: m R N A$ expressions of steroidogenic factor 1 (SF1), steroidogenic acute regulatory protein (StAR), cytochrome

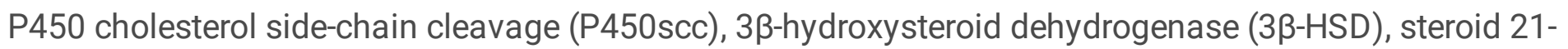
hydroxylase (P450c21) and steroid 11 $\beta$-hydroxylase (P450c21) determined by real-time quantitative polymerase chain reaction (RT-qPCR) after birth, $n=12$, H-L: Protein expressions of SF1 and StAR 
determined by immumohistochemical staining (magnification: $\times 200$ ) and western blot, $\mathrm{n}=3$,

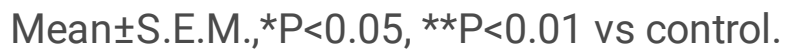
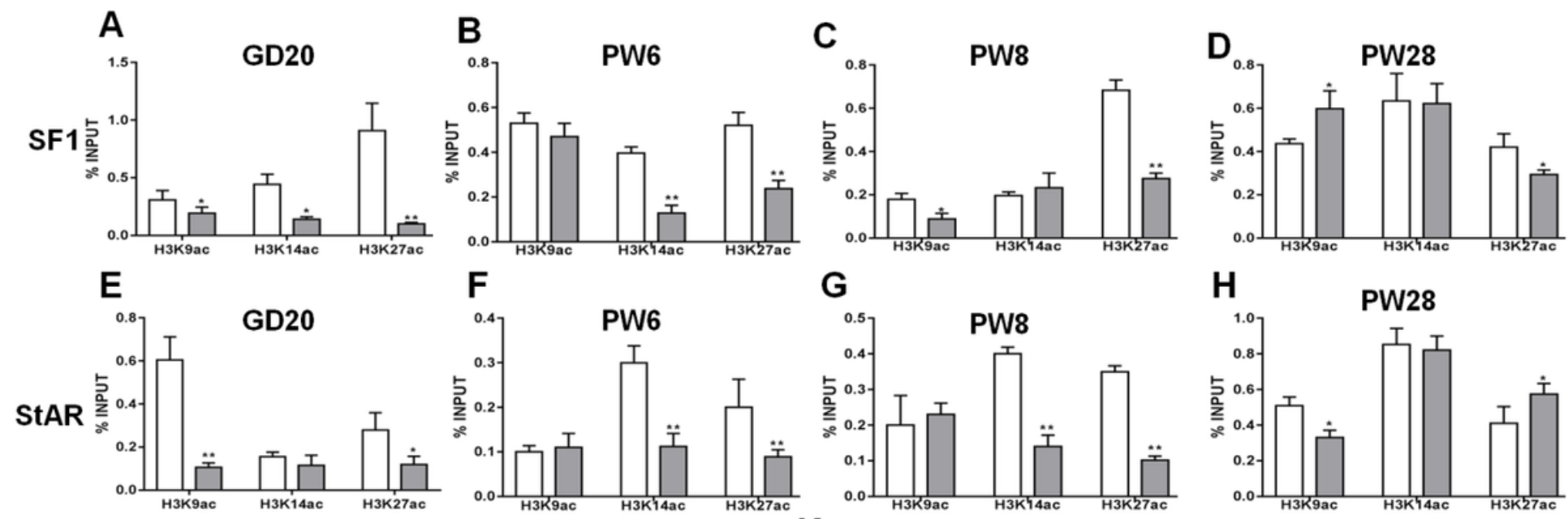

G
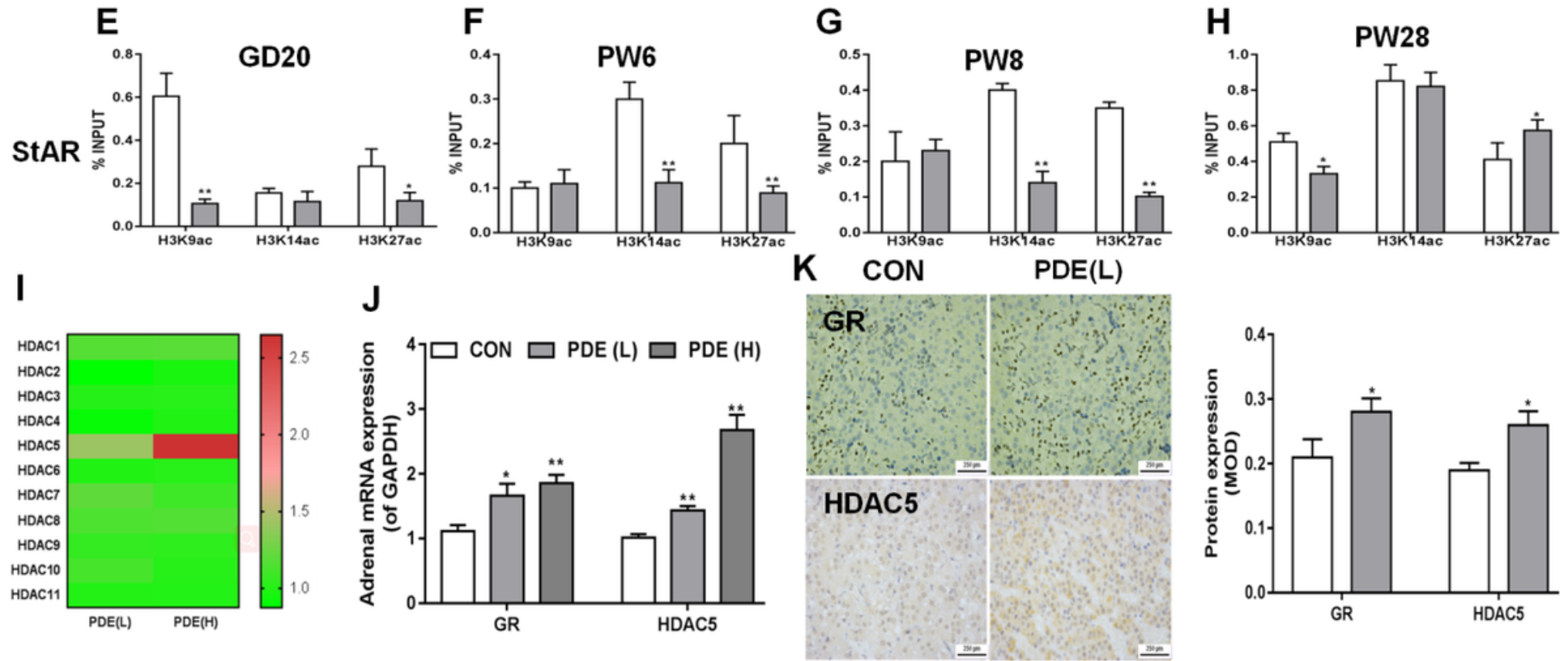

Figure 3

Effects of prenatal dexamethasone exposure (PDE) on expression of adrenal glucocorticoids receptor (GR)/histone deacetylase 5 (HDAC5) and histone acetylation level of steroidogenic factor 1

(SF1)/steroidogenic acute regulatory (StAR) in male offspring rats. A-D: Enrichment of the histone 3 lysine 9 acetylation (H3K9ac), H3K14ac, and H3K27ac levels in SF1 promoter analyzed by chromatin immunoprecipitation-polymerase chain reaction (ChIP-PCR) at gestational day (GD) 0, postnatal week (PW) 6, PW8 and PW28, n=12, E-H: Enrichment of the H3K9ac, H3K14ac, and H3K27ac levels in StAR promoter analyzed by ChIP-PCR at GD0, PW6, PW8 and PW28, n=12, I: real-time quantitative polymerase chain reaction (RT-qPCR) analysis of gene expression of HDACs at GD0, $n=12$. J: mRNA expression of GR and HDAC5 determined by RT-qPCR at GD0, $n=12, K$ : Protein abundance of GR and HDAC5 detected by immumohistochemical staining at GD0 (magnification: $\times 200$ ), $n=3$ (three fetal adrenals from three littermates were pooled for homogenization into one sample). Mean $\pm S$.E.M., ${ }^{*}<<0.05,{ }^{\star}{ }^{*}<<0.01$ vs control. 
A
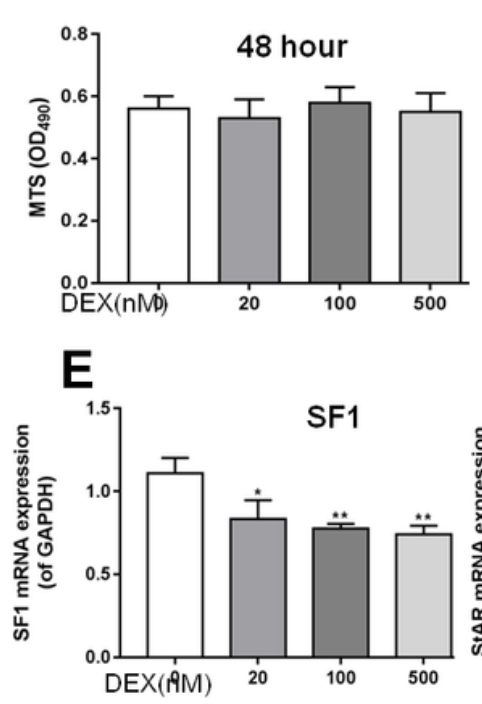

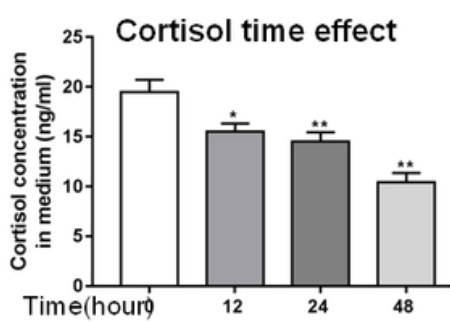

\section{B}

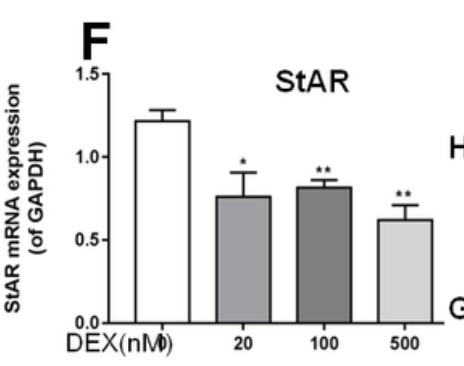

C

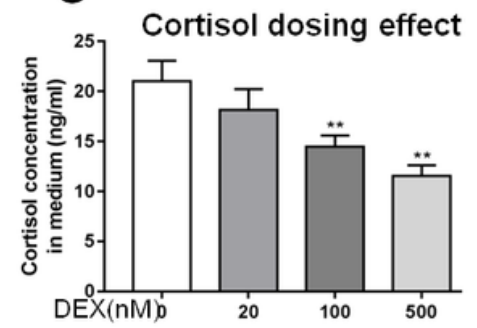

G

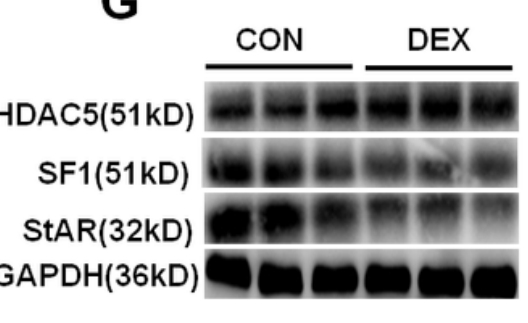

D
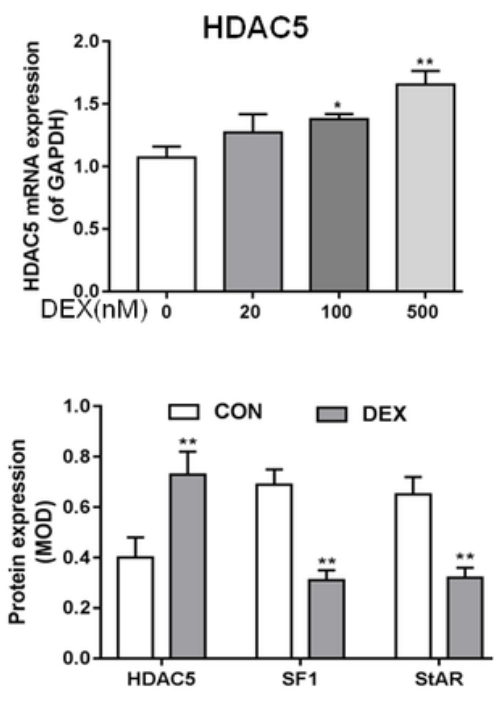

Figure 4

Effects of dexamethasone on steroid synthesis function, expression and activity of glucocorticoids receptor (GR)/histone deacetylase 5 (HDAC5) in H295R cells. A: Concentration-dependent effects of dexamethasone on H295R cell viability by MTS in 48 hour, B,C: Cortisol content in the cell media treated with dexamethasone, D-F: mRNA expression of HDAC5, steroidogenic factor 1 (SF1)/steroidogenic acute regulatory protein (StAR) in H295R cells treated with dexamethasone at the concentration of $0 \sim 500 \mathrm{nM}$, G: Protein expression of HDAC5, SF1 and StAR in H295R cells treated with $500 \mathrm{nM}$ of dexamethasone. Mean \pm S.E.M., $n=3$ for each group. ${ }^{*}<0.05,{ }^{*} P<0.01$ compared with the untreated cells. 

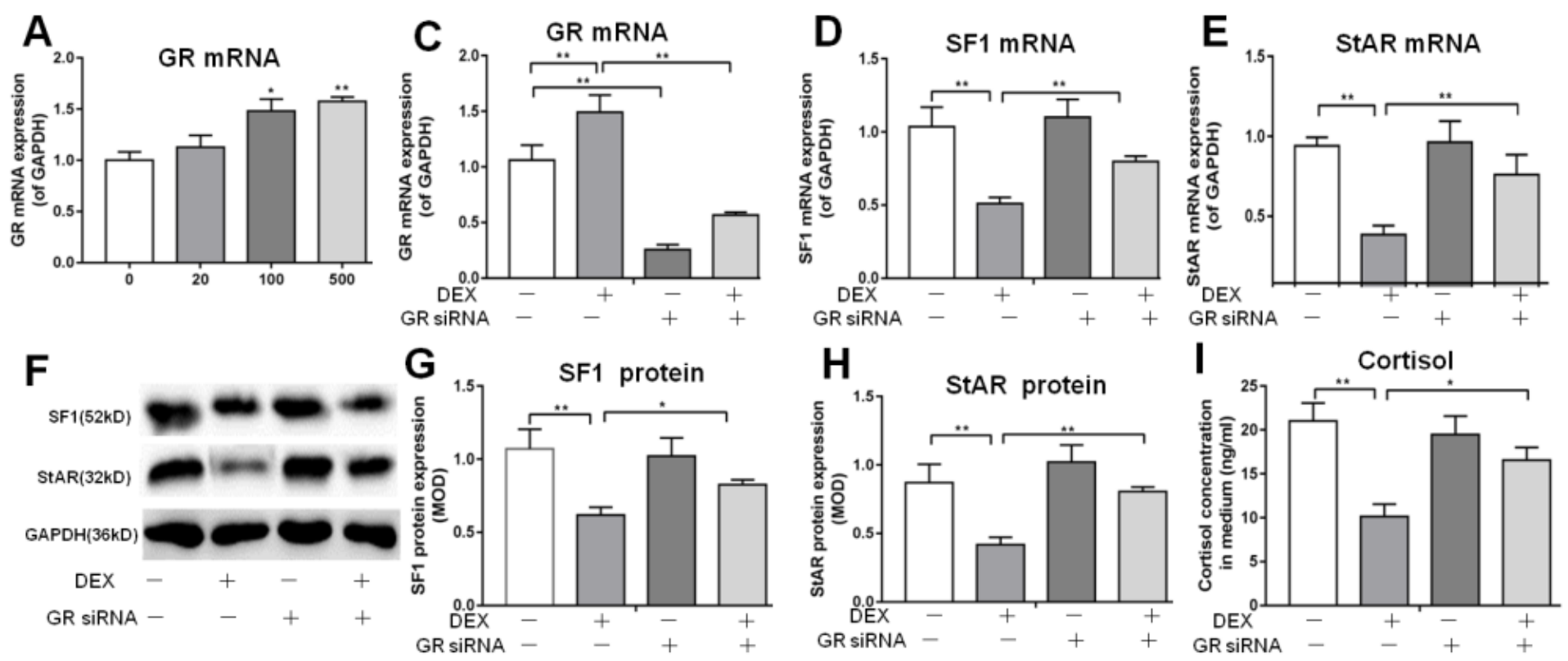

\section{B Cytoplasm Nucleus}

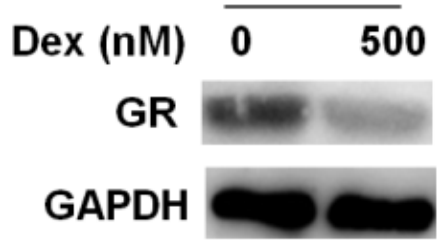

Histone3

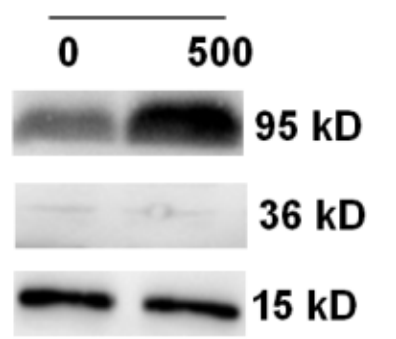

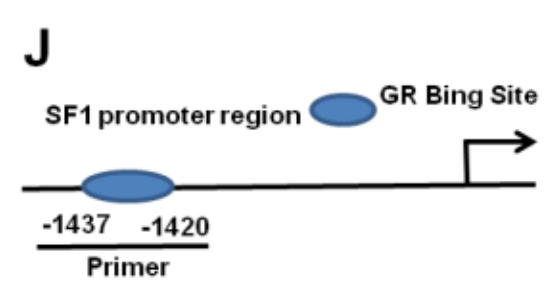

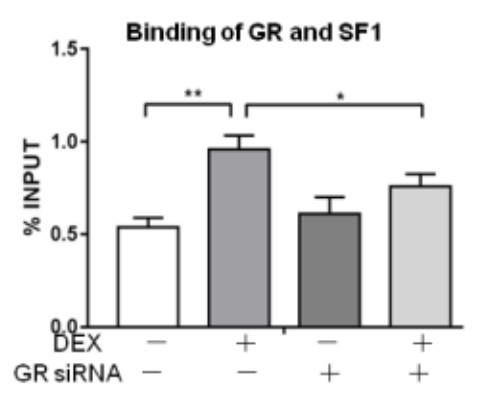

Figure 5

The transcriptional regulatory mechanisms of activated glucocorticoids receptor (GR) by dexamethasone, binding into steroidogenic factor 1 (SF1) and inhibiting adrenal steroid synthesis in H295R cells. A: mRNA expression of GR in H295R cells treated with dexamethasone at the concentration of $0 \sim 500 \mathrm{nM}, \mathrm{B}$ :

Protein abundance of GR in cytoplasm and nucleus in H295R cells treated with $500 \mathrm{nM}$ of dexamethasone, C-E: mRNA expression of GR, SF1 and steroidogenic acute regulatory protein (StAR), F-H: protein expression of SF1 and StAR, I: cortisol content in the cell medium, J: the enrichment of GR at the SF1 promoter. Mean $\pm S$.E.M., $n=3$ for each group. ${ }^{\star} P<0.05,{ }^{*} \mathrm{P}<0.01$ vs control. 

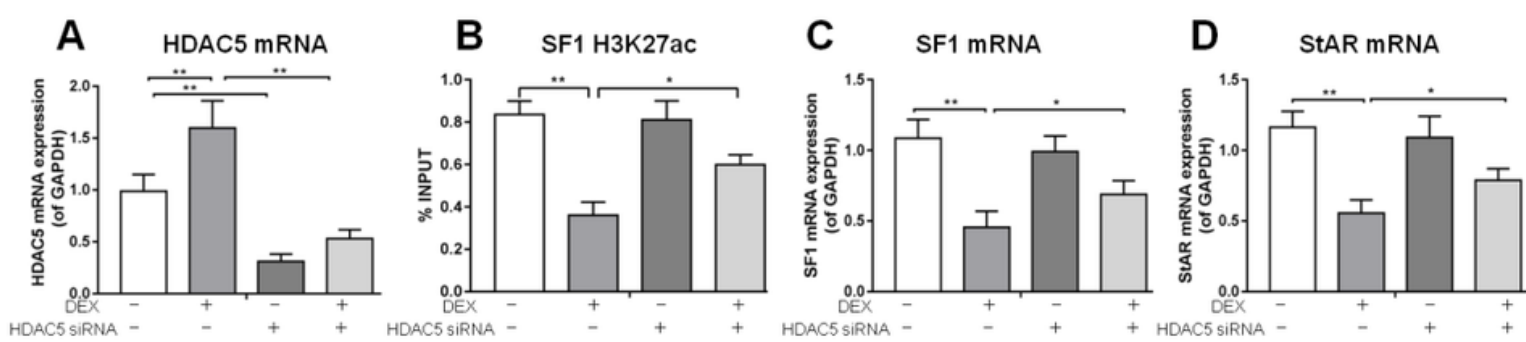

E
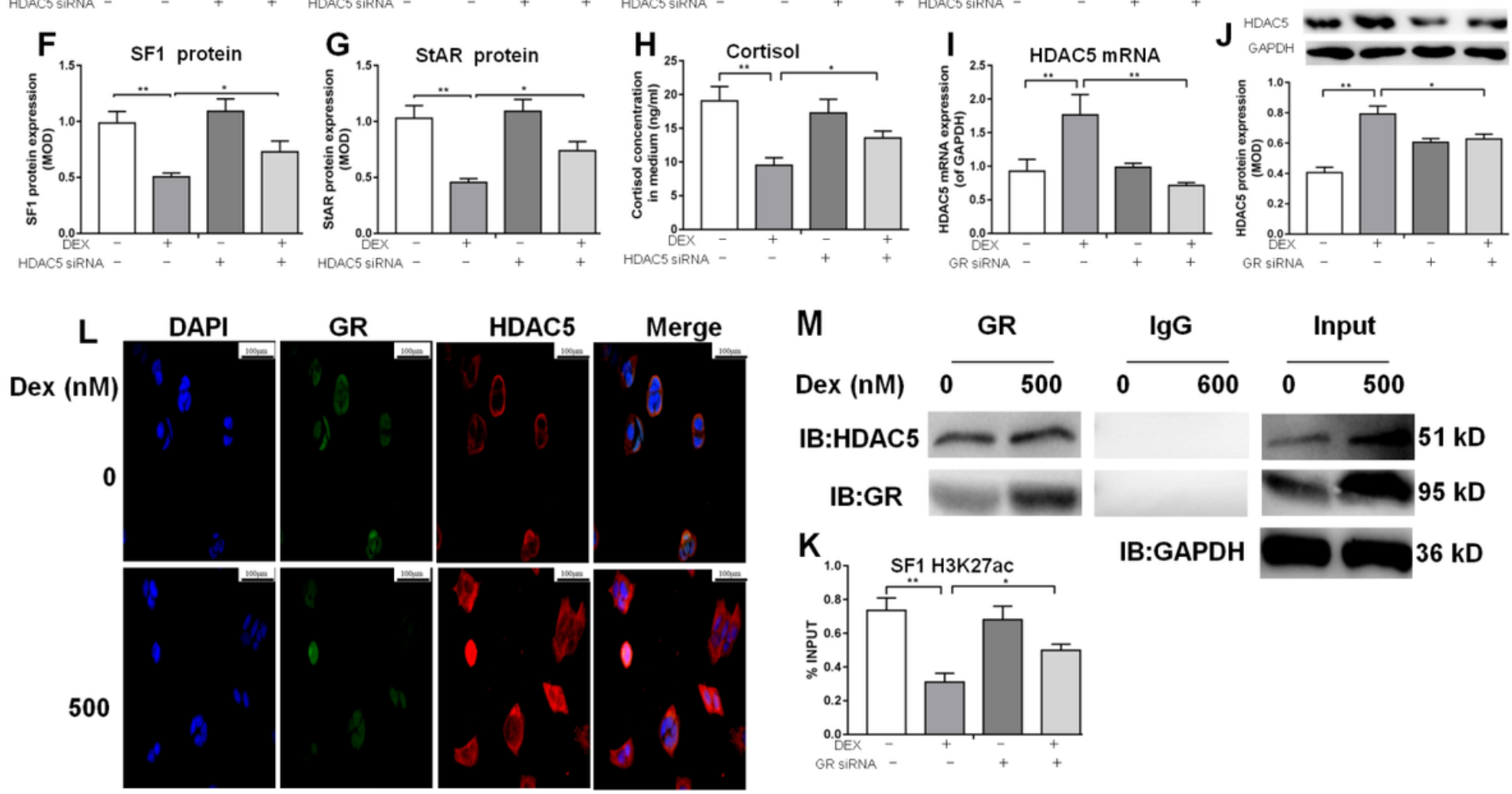

\section{Figure 6}

The epigenetic mechanism of decreased steroidogenic factor 1 (SF1) expression induced by dexamethasone in H295R cells. A: mRNA expression of histone deacetylase 5 (HDAC5), B,K: Enrichment of histone 3 lysine 27 acetylation (H3K27ac) in SF promoter, C,D,l: mRNA expression of SF1, steroidogenic acute regulatory protein (StAR) and HDAC5, E-G,J: protein expression of SF1, StAR and HDAC5, H: cortisol content in the cell medium, L: Co-localization of GR and HDAC5 using immunofluorescence assay in H295R cells treated with $500 \mathrm{nM}$ of dexamethasone, M: Co-Immunoprecipitation (Co-IP) assay of GR and HDAC5 in H295R cells treated with $500 \mathrm{nM}$ of dexamethasone. Mean $\pm S . E . M ., n=3$ for each group. ${ }^{*}<0.05$, $\star * P<0.01$ vs control. 

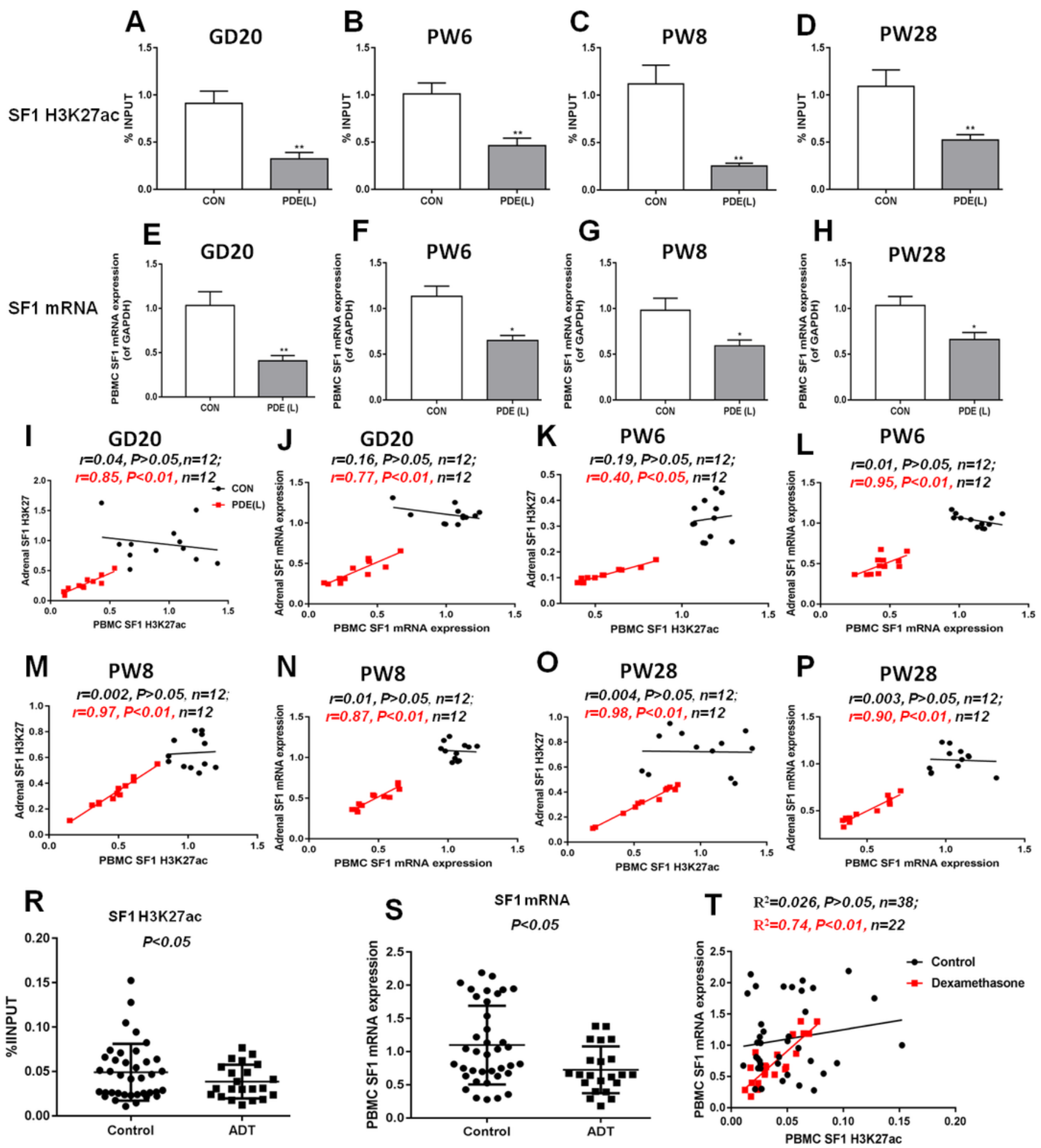

Figure 7

Effects of prenatal dexamethasone exposure (PDE) on the histone 3 lysine 27 acetylation (H3K27ac) level of steroidogenic factor 1 (SF1) promoter and its mRNA expression in peripheral blood mononuclear cell (PBMC) of male offspring rat and neonate. A-D: the H3K27ac level of SF1 in offspring rat adrenal, E-H: mRNA expression of SF1 in offspring rat adrenal, I/K/M/O: the correlation analysis of H3K27ac level of SF1 promoter in offspring rat adrenal and PBMC, J,L,N,P: the correlation analysis of mRNA expression of 
SF1 in offspring rat adrenal and PBMC, R: the H3K27ac level of SF1 promoter in neonate PBMC, S: mRNA expression of SF1 in neonate PBMC, T: the correlation analysis between the H3K27ac level of SF1 promoter and its $m R N A$ expression in neonate PBMC. $n=12$ for each group in rat(one littermate fetal serum was merged into one sample, three pairs fetal adrenals from three littermates were pooled for homogenization into one sample), $n=22$ in control group, $n=38$ in PDE group. ${ }^{*} P<0.05,{ }^{\star} * P<0.01$ vs control.

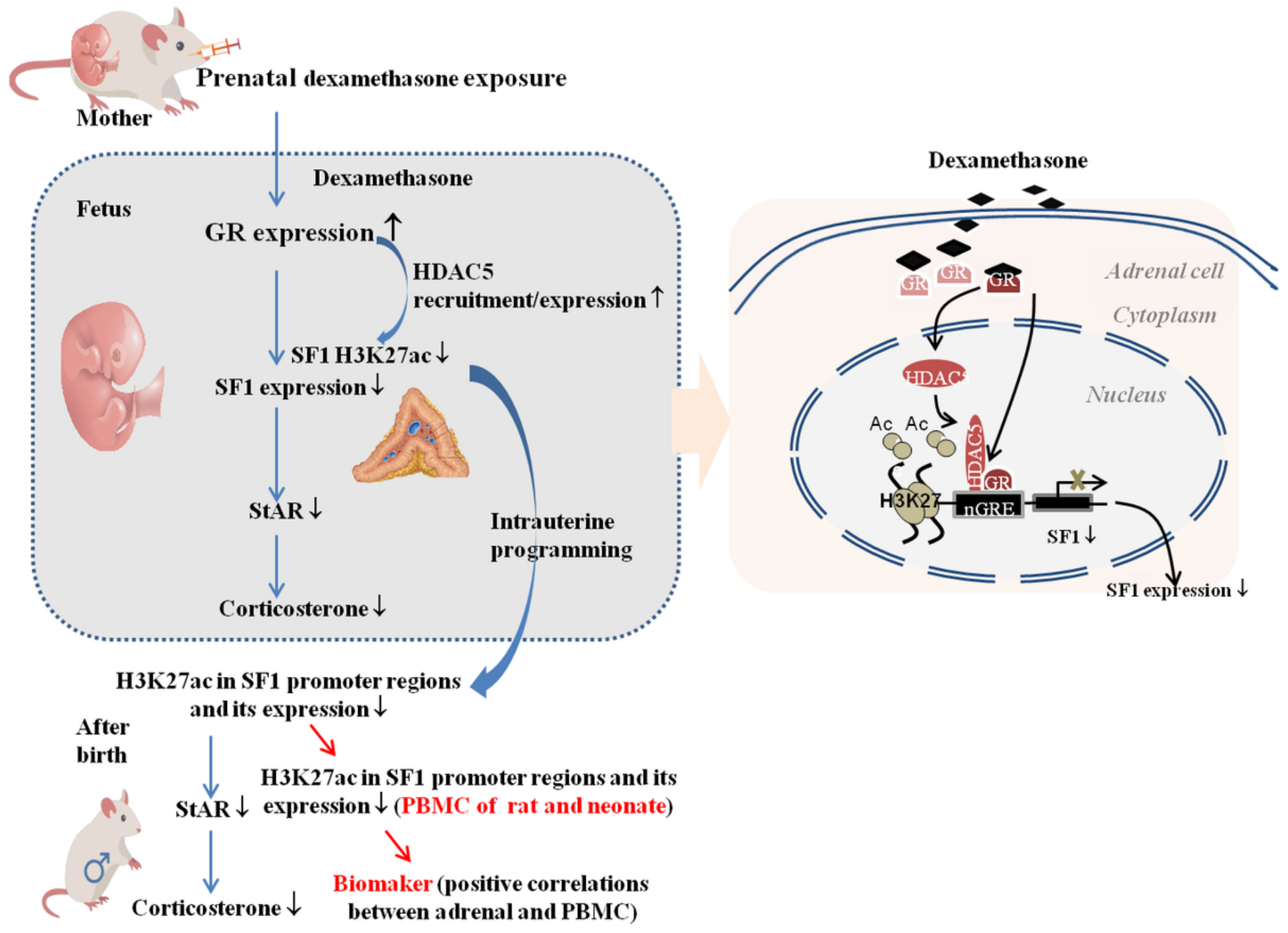

Figure 8

Intrauterine programming mechanism of low adrenal steroidogenesis function induced by prenatal dexamethasone exposure. GR, glucocorticoids receptor, HDAC5, histone deacetylase 5, SF1, steroidogenic factor 1, H3K27ac, histone 3 lysine 27 acetylation, StAR, steroidogenic acute regulatory. 